\title{
Das Retentionsindex-System, seine Anwendung zur Identifizierung von Substanzen und Charakterisierung von flüssigen Phasen ${ }^{*}$ )
}

\section{Teil II. Zusammenhang zwischen Retentionsindex, Struktur und Analysenparametern ${ }^{* *}$ )}

\section{S. Ettre}

The Perkin-Elmer Corporation, Norwalk, Connecticut 06856, USA

Der erste Teil dieser Serie befaßte sich mit der Bedeutung des Retentionsindex und der Art seiner Berechnung. Auf Grund der Tatsache, daß sein Wert nicht von Geräteparametern abhängt, sondern ausschließlich von der stationären (flüssigen) Phase und von der Temperatur, ist der Retentionsindex offensichtlich zur Identifizierung von Substanzen ideal geeignet.

Der Retentionsindex-Bégriff kann jedoch nicht nur für die Identifizierung durch Tabellenvergleich ("table matching") eingesetzt werden, um den Ausdruck von Schomburg und Dielmann [16] zu verwenden, d. h. Vergleich der gemessenen Retentionsindex-Werte mit Datensammlungen. Die Retentionsindex-Werte können auch mit der Struktur der analysierten Substanzen, mit der relativen Retention zweier Gelöster und mit dem Verhältnis zwischen Retentionsverhalten und analytischen Bedingungen wie 2. B. Temperatur in Beziehung gesetzt werden. Diese Beziehungen können als Funktionen verschiedener RetentionsindexInkremente ausgedrückt werden; deshalb wollen wir im vorliegenden zweiten Teil unserer Serie kurz die Bedeutung der verschiedenen Retentionsindex-Inkremente diskutieren.

Es muß erwähnt werden, daß die Literatur, die sich mit diesen Beziehungen befaßt, besonders mit denjenigen zwischen Retentionsindex-Inkrementen und Struktur, sehr ausführlich ist, und wir können deshalb nicht auf Einzelheiten eingehen oder auch nur eine ausführliche Bibliographie zitieren. Die Leser, die sich darüber eingehender informieren wollen, werden auf die Artikeisammlung verwiesen, die kürzlich zu diesem Thema publiziert wurde $[16,23-26]$, und in der auch eine ausfuihrliche Bibliographie enthalten ist.

Unsere Diskussion wird sich auf füf RetentionsindexDifferenzen oder, gemäß der üblichen Bezeichnung, Inkremente konzentrieren. Sie werden nachstehend zusam-

*) Der erste Teil dieser Serie wurde in Chromatographia Heft 12, S. 525 (1973) veröffentlicht.

${ }^{* *}$ )Die Literaturzitate, Gleichungen, Tabellen und Abbildungen sind innerhalb der gesamten Serie durchnumeriert. Lit. [1-22] siehe Teil I. men mit den gegebenenfalls verwendeten Symbolen erläutert:

Die Differenz der Retentions-Indices eines einzigen Gelösten, gemessen auf derselben flüssigen Phase bei zwei verschiedenen Temperaturen $\partial \mathbf{I}$

Die Differenz der Retentions-Indices zweier Gelöster, die zur selben homologen Reihe gehören und sich nur um ein C-Atom unterscheiden, gemessen auf derselben flüssigen Phase bei derselben Temperatur.

Die Differenz der Retentions-Indices zweier Gelöster, wobei eins die zu untersuchende Substanz darstellt und das andere das n-Paraffin mit derselben C-Zahl, gemessen auf einer unpolaren flüssigen Phase bei derselben Temperatur $\mathrm{H}^{\mathrm{NP}}$

Die Differenz der Retentions-Indices eines einzigen Gelösten, gemessen auf zwei verschiedenen flüssigen Phasen, einer polaren und einer unpolaren, bei derselben Temperatur $\Delta \mathrm{I}$

Die Differenz der Retentions-Indices zweier verschiedener Gelöster auf derselben flüssigen Phase bei derselben Temperatur i

Der erste Wert - $\partial \mathrm{I}$ - bezeichnet die Temperaturabhängigkeit des Retentionsindex; die drei nächsten Werte setzen den Retentionsindex in Beziehung zur molekularen Struktur des Gelösten, während sich mit dem letzten Wert - i-der Grad der Auflösung zweier Substanzen auf einer gegebenen Säule erkennen läßt.

\section{Temperaturabhängigkeit des Retentionsindex}

Wir haben schon im ersten Teil dieser Serie erwähnt, daß das Retentionsverhältnis $\mathrm{r}$ temperaturabhängig ist. Diese Abhängigkeit kann durch die folgende Gleichung ausgedrückt werden $[27,28]$ :

$$
\log r=a+\frac{b}{T}
$$

wobei $\mathrm{T}$ die Temperatur in ${ }^{\circ} \mathrm{K}$ ist, während $\mathrm{a}$ und $\mathrm{b}$ Konstanten sind. 
Wir haben in Gl. 12 (Teil I) gesehen, daß der Retentionsindex als Funktion zweier relativer Retentionswerte ausgedrückt werden kann:

$$
I=100 z+100 \frac{\log r_{x / z}}{\log r_{(z+1) / z}}
$$

Natürlich sind beide relativen Retentionen temperaturabhängig und können durch Gl. 14 ausgedrückt werden; nur die Konstanten sind verschieden:

$$
\begin{aligned}
& \log r_{x / z}=a+\frac{b}{T} \\
& \log r_{(z+1) / z}=c+\frac{d}{T}
\end{aligned}
$$

Wenn man diese Gleichungen in Gl. 12 einsetzt, können wir schreiben, daß

$$
\mathrm{I} \propto \frac{\mathrm{aT}+\mathrm{b}}{\mathrm{cT}+\mathrm{d}}
$$

wobei $a, b, c$ und d Konstanten sind.

Eingehende in der Literatur beschriebene Untersuchungen (siehe z. B. $[29,30]$ ) zeigten, daß die tatsächliche Beziehung zwischen Retentionsindex und Säulentemperatur gewöhnlich durch eine Antoine'sche Gleichung beschrieben werden kann:

$$
\mathrm{I}=\mathrm{A}+\frac{\mathrm{B}}{\mathrm{T}+\mathrm{C}}
$$

wobei A, B und C Konstanten sind, abhängig von dem Gelösten und der fliussigen Phase. Trägt man I gegen T auf, so ergibt sich nach Gl. 17 eine Hyperbel, jedoch wurde gezeigt $[12,28]$, daß in dem in der Praxis verwendeten Temperaturintervall die Funktion I gegen $\mathrm{T}$ einen linearen Teil hat, so daß wir schreiben können:

$$
\mathrm{I} \simeq \mathrm{a}^{\prime} \mathrm{T}+\mathrm{b}^{\prime}
$$

wobei a' und b' Konstanten sind. Bei einer unpolaren fliissigen Phase erstreckt sich diese lineare Beziehung gewöhnlich über einen größeren Temperaturbereich als bei einer polaren.

In den meisten Fällen ist $\mathrm{a}^{\prime}>0$, und deshalb erhöht sich der Retentionsindex mit steigender Temperatur; jedoch gibt es auch Fälle, wo das Gegenteil zutrifft.

Auf Grund der in Gl. 18 beschriebenen annähernd linearen Beziehung kann man die Temperaturabhängigkeit des Retentionsindex ausdrücken, indem man das Inkrement für einen gegebenen Temperaturbereich angibt, üblicherweise $10^{\circ} \mathrm{C}$; und dann diesen Wert benutzt, um den Retentionsindex für eine andere Temperatur als die, bei der er bestimmt wurde, zu ermitteln. Wenn z. B. die folgenden Werte [31] für n-Propylacetat angegeben werden:

$$
\mathrm{I}_{80^{\circ} \mathrm{C}}^{\text {Carbowax } 400}=1043 \quad \partial \mathrm{I} / 10^{\circ}=4,25
$$

dann kann man z. B. den Wert bei $60^{\circ} \mathrm{C}$ berechnen als $1043--2 \times 4,25=1034$. Der tatsächliche Wert ist 1035 .

Hier sind zwei Bemerkungen angebracht. Zunächst einmal sollte man nicht zu viel extra- oder interpolieren,

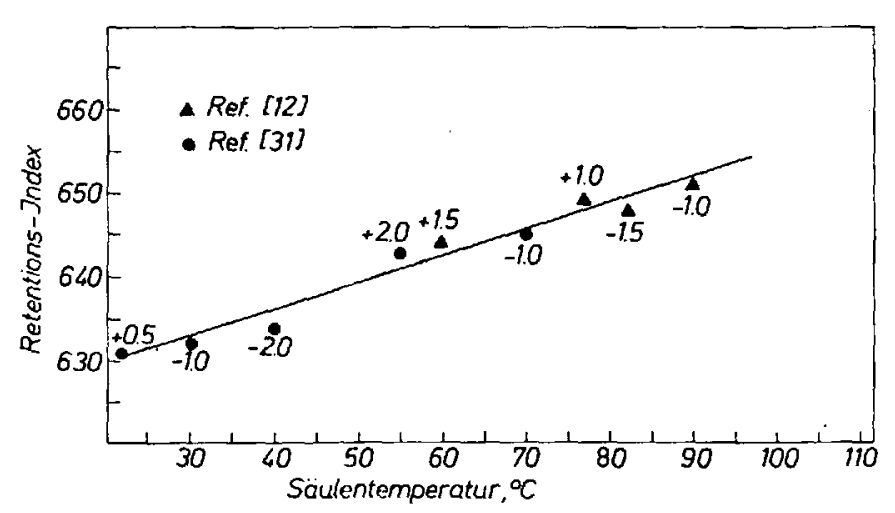

- Fig. 4

Retentionsindex-Werte von Benzol auf Squalan, aufgetragen gegen die Temperatur. Die Ziffern bezeichnen die Abweichungen der tatsächlichen Werte (in Index-Einheiten) von der Geraden, dje durch diese Punkte gelegt wurde.

denn die Beziehung I gegen $T$ ist für einen weiteren Temperaturbereich nicht mehr linear, und bei polaren Phasen muß man besonders vorsichtig sein. Zweitens ist es besser, die Werte $\partial \mathrm{I} / \partial \mathrm{T}$ graphisch zu ermitteln, wobei Ungenauigkeiten in der Bestimmung vermieden werden können.

Zum Beispiel sind in Fig. 4 die Retentionsindex-Werte für Benzol auf Squalan aus zwei Literaturangaben aufgetragen, wobei auch die Abweichungen der einzelnen Messungen von der linearen Funktion angegeben sind [12]. Die eingezeichnete Gerade bildet die beste Annäherung an die gemessenen Werte; deshalb sollten wir den Wert von $\partial \mathrm{I} / \partial \mathrm{T}$ aus dieser Funktion ermitteln. Zum Beispiel können wir die folgenden Werte ablesen:

$$
\mathrm{I}_{30^{\circ}}=633 \quad \mathrm{I}_{60^{\circ}}=643 \quad \mathrm{I}_{90^{\circ}}=652
$$

Die Differenz für ein Intervall von $60^{\circ} \mathrm{C}$ ist $652-633$ $=19$ Indexeinheiten; daher ist $\partial \mathrm{I} / 10^{\circ}$ gleich 3,2 .

Bei einer Aufstellung von Retentionsindices würden wir deshalb die Werte von Benzol auf Squalan wie folgt angeben:

$$
\mathrm{I}_{60^{\circ} \mathrm{C}}^{\text {Squatan }}=643 \quad \partial \mathrm{I} / 10^{\circ} \mathrm{C}=3,2
$$

und aus diesen Werten den Retentionsindex bei anderen Temperaturen berechnen. In Tabelle IV sind die gemessenen Werte aufgefuihrt, die in Fig. 4 aufgetragen wurden, sowie die auf diese Weise berechneten Werte. Wie man sieht, ist die Übereinstimmung ziemlich gut, sie bewegt sich innerhalb von 3 Index-Einheiten, und im Augenblick ist das etwa die Genauigkeit, die man bei Verwendung von Daten aus verschiedenen Laboratorien erwarten kann.

Natürlich kann man auch die graphische Darstellung yon I gegen $T$ verwenden, um die Temperaturabhängigkeit des Retentionsindex anzugeben; die Fig. 5 und 6 zeigen solche Funktionen für eine Anzahl von Kohlenwasserstoffen auf der flüssigen Phase Squalan [12].

Abgesehen von der Möglichkeit, den RetentionsindexWert einer gegebenen Substanz bei jeder beliebigen Temperatur direkt ablesen zu können, können solche Darstellungen auch auf zweierlei Weise zusätzlich von Nutzen 
Tabelle IV. Retentionsindex-Werte von Benzol auf Squalan

\begin{tabular}{c|c|c|c}
\hline $\begin{array}{c}\text { Temperatur } \\
{ }^{\circ} \mathrm{C}\end{array}$ & $\begin{array}{c}\text { Literatur- } \\
\text { angaben }^{\mathbf{a}}\end{array}$ & $\begin{array}{c}\text { Berechnete }^{\mathrm{Werte}}{ }^{\mathrm{b}} \\
\text { We }^{2}\end{array}$ & Differenz \\
30 & 631 & 631 & 0 \\
40 & 632 & 633 & +1 \\
55 & 634 & 637 & +3 \\
60 & 643 & 641 & -2 \\
70 & $644^{*}$ & 643 & -1 \\
77 & 645 & 646 & +1 \\
82 & $649^{*}$ & 648 & -1 \\
90 & $648^{*}$ & 650 & +2 \\
\hline
\end{tabular}

a Die Werte mit einem Stern sind der Lit. [12] entnommen, während die anderen von Lit. [31] stammen.

b

unter Verwendung von $\mathrm{I}_{60^{\circ}}=643$ und $\partial \mathrm{I} / 10^{\circ}=3,2$

sein: man kann schnell abschätzen, welches die günstigste Temperatur zur Trennung einer gegebenen Mischung ist; darüberhinaus geben sie zusätzliche Informationen für die qualitative Identifizienung bestimmter Verbindungen im Chromatogramm eines komplexen Gemisches.

Die Möglichkeit zur Abschätzung der geeigneten Analysentemperatur läßt sich am besten durch ein Beispiel illustrieren. Nehmen wir an, daß wir ein Gemisch aus den folgenden drei Komponenten vorliegen haben:

\section{Methylcyclohexan \\ 2,4-Dimethylhexan \\ 2,5-Dimethylhexan.}

Aus Fig. 6 ergibt sich, daß man dieses Gemisch weder bei $60^{\circ} \mathrm{C}$ noch bei $85^{\circ} \mathrm{C}$ analysieren sollte, denn bei diesen Temperaturen wird Methylcyclohexan zusammen mit einem der beiden Isoparaffine eluiert. Deshalb sollte eine Temperatur von etwa $40-50^{\circ} \mathrm{C}, 75^{\circ} \mathrm{C}$ oder $100^{\circ} \mathrm{C}$ gewählt werden.

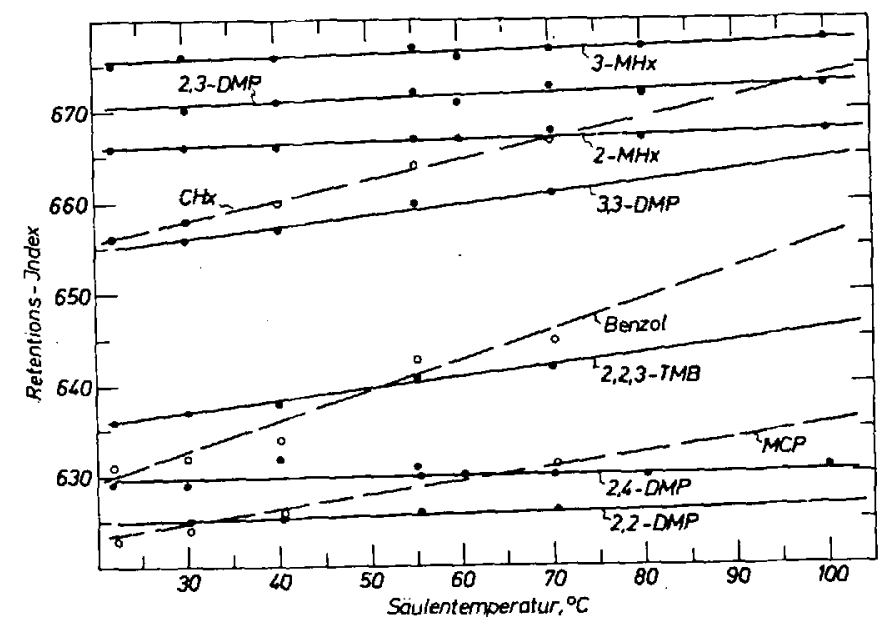

- Fig. 5

Retentionsindex-Werte von Kohlenwasserstoffen auf Squalan, aufgetragen gegen die Temperatur. Index-Bereich: 620-680.

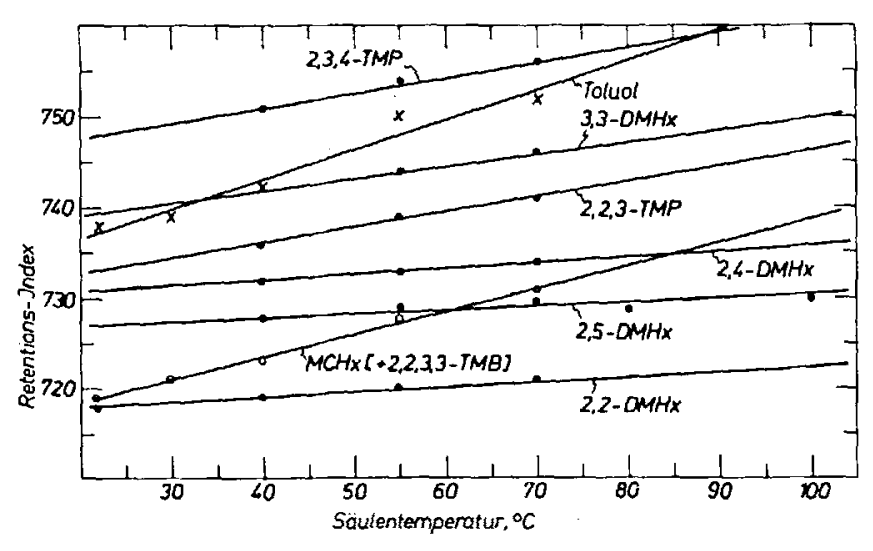

- Fig. 6

Retentionsindex-Werte von Kohlenwasserstoffen auf Squalan, aufgetragen gegen die Temperatur. Index-Bercich:710-760. Abkürzungen in den Fig. 5-6:

TMB = Trimethylbutan

DMP = Dimethylpentan

TMP = Trimethylpentan

$\mathrm{MHx}=$ Methylhexan

DMHx $=$ Dimethylhexan

MCP = Methylcyclopentan

$\mathrm{CHx}=$ Cyclohexan

$\mathrm{MCHx}=$ Methylcyclohexan

Die Möglichkeit, solche Funktionen als zusätzliche Informationen zur qualitativen Identifizierung zu verwenden, basiert auf der unterschiedlichen Temperaturabhängigkeit der Substanzklassen, d. h. auf dem verschiedenen Anstiegsgrad der einzelnen Funktionen. Zum Beispiel sieht man, daß auf Squalan diejenigen Isoparaffine einen stärkeren Anstieg zeigen, die mehr Seitengruppen haben; man vergleiche 3-Methylhexan mit 3,3-Dimethylpentan oder 2,4-Dimethylhexan mit 2,2,3-Trimethylpentan. Die Funktionen von Cycloparaffinen und Aromaten (und ,sehr stark verzweigten" Isoparaffinen) haben eine so starke Steigung, daß diese Funktionen tatsächlich diejenigen der Isoparaffine überschneiden; das kann zur Folge haben, daß in einem komplexen Gemisch die Peaks von Cycloparaffinen und Aromaten im Verhältnis zu den Peaks von Iso- (und natïrlich Normal-) Paraffinen ihre Reihenfolge vertauschen können, wenn die Analyse bei verschiedenen Temperaturen durchgeführt wird. Nehmen wir z. B. das vorher erwähnte Beispiel, eine Dreikomponentenmischung aus Methylcyclohexan (MCHx), 2,4- und 2,5-Dimethylhexan (DMHx). Aus Fig. 6 läßt sich leicht folgern, daß die Reihenfolge der Peaks von der Analysentemperatur abhängt:

$$
\begin{aligned}
& \text { unterhalb } 60^{\circ} \mathrm{C}: \mathrm{MCHx} \text {-2,5-DMHx-2,4-DMHx } \\
& 60-85^{\circ} \mathrm{C} \text { : 2,5-DMHx-MCHx - 2,4-DMHx } \\
& \text { über } 85^{\circ} \mathrm{C} \text { : 2,5-DMHx-2,4-DMHx-MCHx. }
\end{aligned}
$$

Fig. 7 zeigt die drei Chromatogramme, die bei den jeweiligen Temperaturen von 45,74 und $95^{\circ} \mathrm{C}$ registriert wurden, und die diese Verschiebung der Peaks illustrieren.

Eine praktische Anwendung dieser Peak-Verschiebung ist die versuchsweise Identifizierung solcher Komponenten in einem komplexen Kohlenwasserstoff-Gemisch. Man muß 

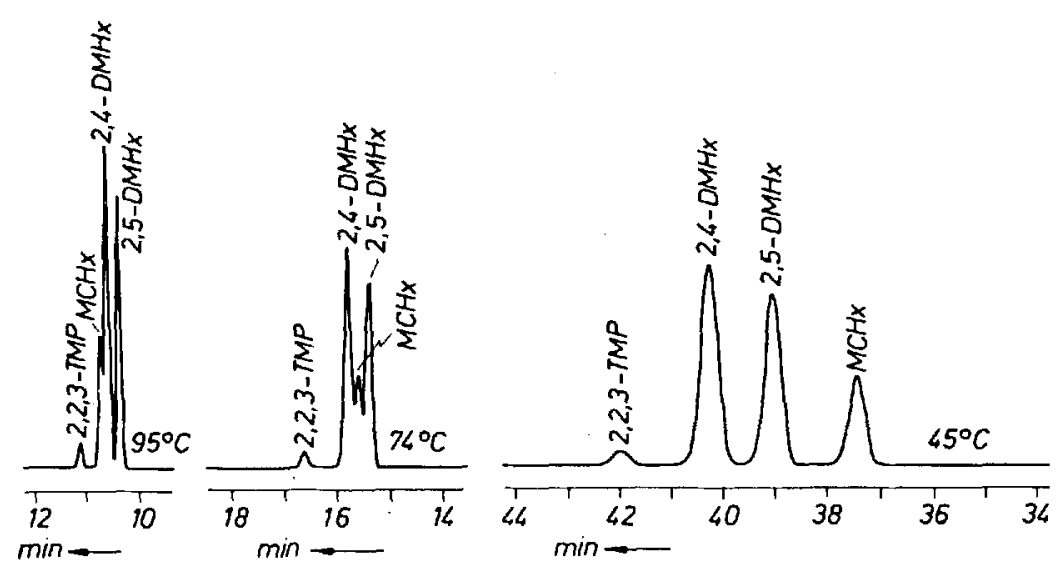

- Fig. 7

Chromatogramm eines Gemisches von Methylcyclohexan (MCHx), 2,5-Dimethylhexan (2,5-DMHx), 2,4-Dimcthylhexan (2,4-DMHx) und 2,2,3-Trimethylpentan (2,2,3-TMP) auf Squalan bei drei verschiedenen Temperaturen. Säule: $150 \mathrm{ft}$ x $0,50 \mathrm{~mm}$ $\phi_{\mathrm{i}}$ gepackte Kapillarsäule mit Squalan als flüssige Phase. Mittlere lineare Strömungsgeschwindigkeit des Trägergases (He): $20 \pm 1 \mathrm{~cm} / \mathrm{sec}$. nur die Probe auf Squalan (oder einer anderen unpolaren Phase) bei drei geeigneten Temperaturen analysieren, die je nach dem Bereich der Probe ausgewählt werden, und dann prüfen, welche Peaks sich im Verhältnis zu den anderen wesentlich verschoben haben. Hieraus kann mit einiger Sicherheit geschlossen werden, daß diese Peaks Cycloparaffine und/oder aromatische Kohlenwasserstoffe darstellen.

\section{Beziehung zwischen Retentionsindex und Molekül- Struktur}

Der Retentionsindex kann als eine additive Funktion betrachtet werden. Daher sind Retentionsindex-Inkremente - d. h. die Unterschiede zwischen zwei Retentionsindex-Angaben - charakteristisch für die besonderen Gruppen oder Wechselwirkungen, auf die sie sich beziehen.

\section{Inkrement zur Kennzeichnung des Anstiegs des Reten- tionsindex bei einer homologen Reihe}

Das einfachste Retentionsindex-Inkrement bezieht sich auf Glieder einer homologen Reihe, die auf derselben flüssigen Phase (und bei derselben Temperatur) analysiert wurden. Da die Retentionsindex-Skala dem 100-fachen Wert der C-Zahl der n-Paraffine äquivalent ist (z. B. 600 für n-Hexan, 700 für n-Heptan etc.), ist leicht einzusehen, daß die Retentionsindex-Werte zweier Glieder einer homologen Reihe, die sich nur um eine $\mathrm{CH}_{2}$-Gruppe in der Hauptkette unterscheiden, einen Unterschied von 100 Indexeinheiten aufweisen werden. Anders gesagt, wenn wir die Retentionsindices der beiden aufeinanderfolgenden Glieder einer homologen Reihe mit $I_{z}$ und $I_{(z+1)}$ bezeichnen, dann ist

$$
I_{(x+1)}-I_{z}=100
$$

Diese Gleichung gilt nicht, wenn $z$ geringer als 4 oder 5 ist, wegen der bekannten Krümmung der Funktion $\log t_{R}^{\prime}$ gegen Kohlenstoffzahl bei den ersten Gliedern einer homologen Reihe [26]. In der Praxis kann auch im Bereich z $>5$ eine gewisse Abweichung beobachtet werden, aber das ist meistens auf experimentelle Fehler zurückzuführen.
Die Gültigkeit der Gleichung 19 wird durch Fig. 8 illustriert, in der die Funktionen fur eine Anzahl yon homologen Alkan-Reihen aufgetragen sind, die auf Squalan analysiert wurden, und durch Fig. 9, in der solche Funktionen für einige Reihen polarer Verbindungen auf polaren flüssigen Phasen gezeigt sind. Die Angaben für Fig. 8 sind der Ref. [31] entnommen, während diejenigen für Fig. 9 aus der ASTM Datensammlung [13] stammen.

Die praktische Bedeutung der Gl. 19 besteht darin, daß man in dem Chromatogramm eines komplexen Gemisches mit einiger Genauigkeit die Peaks auffinden kann, die den Gliedern gewisser homologer Reihen entsprechen, selbst wenn nur eine begrenzte Zahl von Angaben vorhanden ist.

\section{Inkrement zur Kennzeichnung der Wechselwirkung einer funktionellen Gruppe in einem Molekül im Vergleich zum Stamm-Kohlenwasserstoff}

Wenn wir auf einer unpolaren flüssigen Phase eine Verbindung analysieren, die eine funktionelle Gruppe $\mathrm{R}$ besitzt, und gleichzeitig das entsprechende n-Paraffin mit der gleichen C-Zahl wie die zu untersuchende Substanz, dann wird der Unterschied in den Retentionsindex-Werten mit dem Einfluß der betreffenden funktionellen Gruppe auf die selektive Verzögerung in Beziehung stehen. Wenn also solche Retentionsindex-Inkremente vorliegen, dann kann der Retentionsindex einer Verbindung vorausgesagt werden.

Nehmen wir zum Beispiel die beiden folgenden Verbindungen [16]:

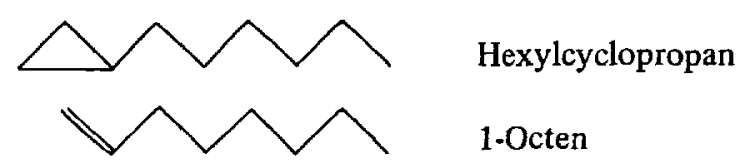

Die jeweiligen Retentionsindex-Werte* bei $80^{\circ} \mathrm{C}$ auf Squalan sind 913,0 und 782,7. Die Stamm-n-Paraffine sind $n-N o n a n(I=900)$ und n-Octan $(I=800)$; also sind

* In der Originalpublikation [16] wurden dic Werte mit zwei Dezimalstellen angegeben; wir rundeten sie hier auf nur eine Stelle nach dem Komma. 


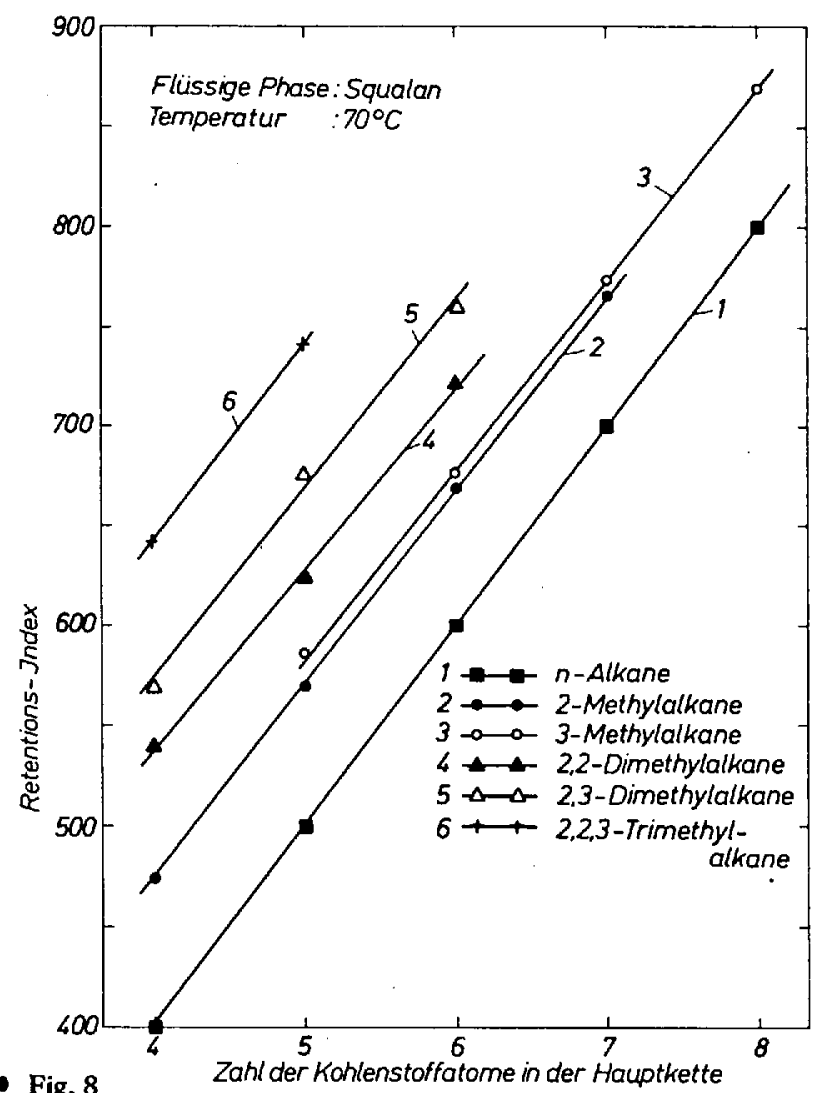

- Fig. 8

Retentionsindices verschiedener homologer Paraffin-Reihen, aufgetragen gegen die C-Zahl der Hauptkette, auf der flüssigen Phase Squalan.

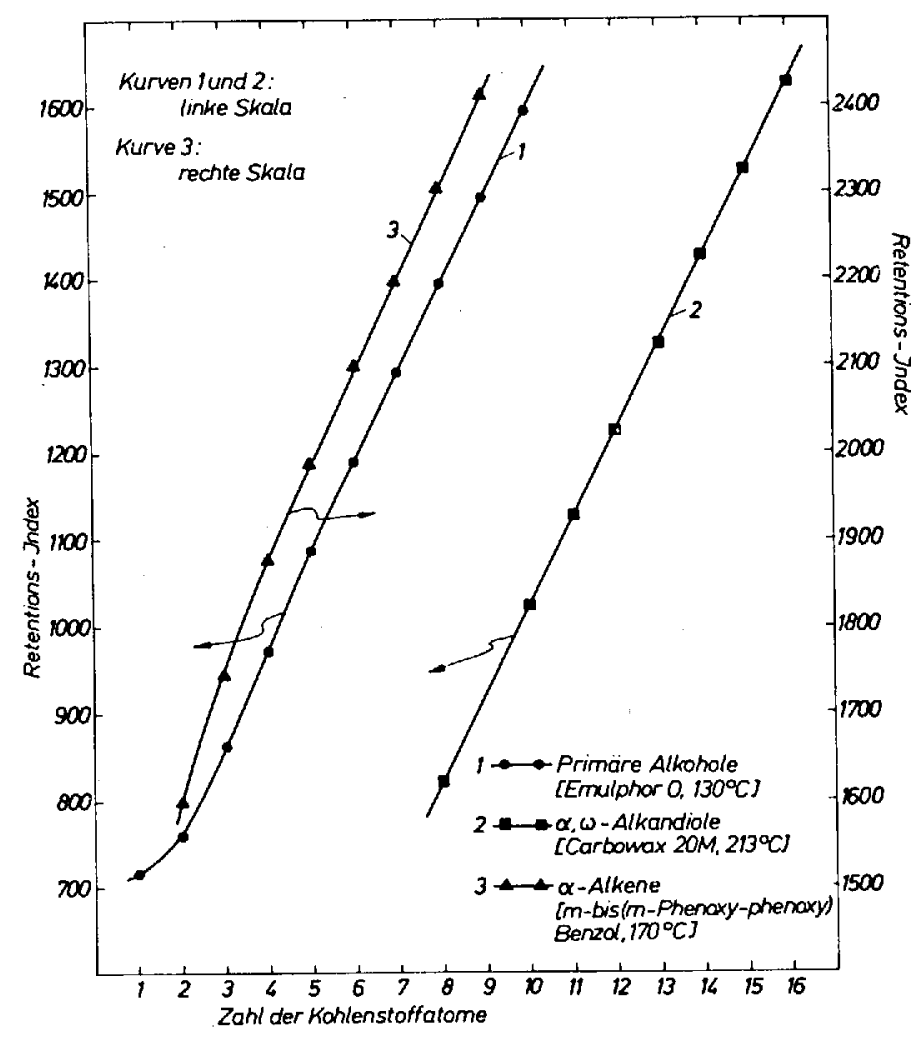

- Fig. 9

Retentionsindices von homologen Reihen polarer Verbindungen, aufgetragen gegen die C-Zahl, auf polaren flüssigen Phasen. die Inkremente der funktionellen Gruppen $+13,0$ und $-17,3$. Nun wollen wir den Retentionsindex der folgenden Verbindung berechnen:

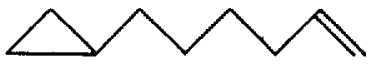

5-Hexenyl-cyclopropan.

Wenn wir den Beitrag der funktionellen Gruppen als additiv annehmen, dann würde das Inkrement für diese Verbindung $+13,0-17,3=-4,3$ betragen. Deshalb beträgt der berechnete Retentionsindex für diese Verbindung $900-4,3=895,7$. Der tatsächlich gemessene Wert beträgt 895,3 , er zeigt also eine ausgezeichnete Ubereinstimmung und beweist die Gültigkeit derartiger Berechnungen. Als weiteres Beispiel berechnen wir den Retentionsindex von 1,7-Octadien. Da das Inkrement für eine Doppelbindung - 17,3 betrug, müßte es das Doppelte, nämlich - 34,6, für zwei betragen; der berechnete Retentionsindex-Wert wäre also $800-34,6=765,4$. Der gemessene Wert ist 765,0 ; auch hier ist die Ubereinstimmung ausgezeichnet.

Das Problem bei solchen Berechnungen liegt darin, daß bei multifunktionellen Verbindungen eine ,Wechselwirkung" zwischen den beiden funktionellen Gruppen auftritt, wenn die funktionellen Gruppen nahe zusammenstehen. Dann entspricht das kombinierte Inkrement nicht mehr der Summe der beiden einzelnen Inkremente. In solchen Fällen kann die Berechnung präzisiert werden, wenn man nach Schomburg vom Abstand abhängige Inkremente zweiter Ordnung heranzieht, die diese ,Wechselwirkung" kennzeichnen [16].

Mathematisch kann der Wert des Inkrements $\mathrm{H}^{\mathrm{NP}}$ für eine funktionelle Gruppe wie folgt ausgedrückt werden:

$$
H^{N P}=I^{N P}(c)-I^{N P}(p)
$$

wobei $\mathrm{I}^{\mathrm{NP}}(\mathrm{c})$ und $\mathrm{I}^{\mathrm{NP}}(\mathrm{p})$ die jeweiligen RetentionsindexWerte der Verbindung bzw. des entsprechenden n-Faraffins auf einer unpolaren Phase darstellen.

Es sollte erwähnt werden, daß das n-Paraffin mit derselben C-Zahl jedem der beiden für die Berechnung des Retentionsindex verwendeten angrenzenden n-Paraffine gleich sein kann. Zum Beispiel enthalten sowohl Hexylcyclopropan $(I=913,0)$ und 5-Hexenylcyclopropan $(\mathrm{I}=895,3)$ jeweils 9 C-Atome im Molekül, und daher ist das entsprechende n-Paraffin n-Nonan. Während jedoch für Hexylcyclopropan n-Nonan das n-Paraffin mit z C-Atomen darstellt, ist es für 5-Hexenylcyclopropan das n-Paraffin mit $z+1$ C-Atomen. Anders gesagt, $I^{N P}(p)$ kann entweder größer oder kleiner als $\mathrm{I}^{\mathrm{NP}}$ (c) sein, und deshalb kann der Wert von $\mathrm{H}^{\mathrm{NP}}$ entweder ein positives oder ein negatives Vorzeichen haben.

Obwohl er theoretisch nicht darauf beschränkt ist, ist der Gebrauch solcher Inkremental-Werte zur Vorhersage des Retentionsindex praktisch nur für Kohlenwasserstoffe möglich, da diese Verbindungen vorzugsweise auf unpolaren flüssigen Phasen analysiert werden. In dieser Hinsicht führten Takács et al. [32, 33] ausführliche Untersuchungen über die Möglichkeit der Berechnung des Retentions- 
Tabelle V. Ergebnisse für die vier isomeren Butylalkohole

\begin{tabular}{|c|c|c|c|c|c|}
\hline \multirow[b]{2}{*}{ Peak } & \multicolumn{3}{|c|}{ Gemessener Retentionsindex } & \multirow[b]{2}{*}{$\begin{array}{c}\Delta \mathrm{I} \\
\text { berechnet }\end{array}$} & \multirow[b]{2}{*}{ Identifizierung } \\
\hline & Apiezon $\mathbf{L}$ & Emulphor O & $\Delta \mathrm{I}$ & & \\
\hline A & 472 & 758 & 286 & 282 & $\begin{array}{l}\text { 2-Methyl- } \\
\text { propanol-2 }\end{array}$ \\
\hline B & 553 & 871 & 318 & 320 & Butanol-2 \\
\hline C & 575 & 929 & 354 & 358 & $\begin{array}{l}\text { 2-Methyl- } \\
\text { propanol-1 }\end{array}$ \\
\hline $\mathrm{D}$ & 606 & 973 & 367 & 373 & Butanol-1 \\
\hline
\end{tabular}

a gemessen bei $130^{\circ} \mathrm{C}$

index von Kohlenwasserstoffen durch, basierend auf der additiven Wirkung des Beitrages verschiedenartiger funktioneller Gruppen und Bindungen im Molekül unter Berücksichtigung ihrer. Wechselwirkungen. Sie bewiesen, daß solche Berechnungen mit guter Richtigkeit möglich sind, und in jüngerer Zeit begannen sie auch, sie auf andere Arten von Gelösten und von flüssigen Phasen auszudehnen [34]; jedoch sind dazu viele experimentelle Werte erforderlich, und die Berechnung ist langwierig. Ein etwas anderes Verfahren, das jedoch ebenfalls die additive Wirkung der Inkremental-Werte heranzog und die Wechselwirkung der einzelnen Gruppen berücksichtigte, wurde von $\mathrm{Ca}$ stello et al. [35] zur Vorhersage des Retentionsindex von Isoparaffinen verwendet. Alle diese Untersuchungen zeigten die solide Basis des Retentionsindex-Konzepts; wegen der großen Anzahl der erforderlichen Werte und wegen der ziemlich komplizierten für die Berechnung verwendeten Gleichungen wird es jedoch noch einige Zeit dauern, bis ihr praktischer Nutzen erwiesen ist.

Inkrement zur Kennzeichnung der Wechselwirkung zwischen funktioneller Gruppe und flüssiger Phase

Diese Art von Inkremental-Werten wurde von Kováts in seinen grundsätzlichen Arbeiten eingeführt [3-6]. Dieses Inkrement ergibt sich aus den Retentionsindices einer Verbindung auf zwei verschiedenen flüssigen Phasen, von denen eine unpolar ist.

$$
\Delta \mathrm{I}=\mathrm{I}^{\text {polar }}-\mathrm{I}^{\text {unpolar }}
$$

Ähnlich dem zuvor diskutierten Wert ist die Indexdifferenz abhängig von der funktionellen Gruppe und ihrer Stellung im Molekül.

Bei einer unpolaren Phase werden die Gelösten etwa in der Reihenfolge ihrer Siedepunkte eluiert, während die Retention in einer polaren Phase zusätzlich auf der Wechselwirkung zwischen den aktiven Gruppen im Molekül des Gelösten und denjenigen der flüssigen Phase beruht. Der Wert von $\Delta \mathrm{I}$ wird also charakteristisch für den $U n$ terschied dieser Wechselwirkungen sein.

Kováts hat gezeigt [3-6], daß man aus der Retention auf den beiden Phasen die individuellen Inkremental-Werte für verschiedene charakteristische Gruppen oder Zonen im Molekül des Gelösten ermitteln kann, die den beobachteten $\Delta \mathrm{I}$-Wert für das betreffende Gelöste additiv beeinflussen. Wenn man also die individuellen InkrementalWerte kennt, dann kann man den für ein gegebenes Gelöstes erwarteten $\Delta \mathrm{I}-$ Wert berechnen. Auf diese Weise kann man durch Vergleich des gemessenen $\Delta \mathrm{I}$-Wertes mit den für verschiedene mögliche Strukturen berechneten Werten die Identität eines bestimmten Peaks ermitteln.

Es ist einfacher, diese Möglichkeit an konkreten Beispielen zu demonstrieren, als sie in allgemeinen Begriffen zu diskutieren. Unser erstes Beispiel behandelt die vier isomeren Butylalkohole. Wir analysierten ein Gemisch davon auf einer Säule mit Apiezon $L$ und einer anderen mit Emulphor $\mathrm{O}$ und berechneten die $\Delta \mathrm{I}$-Werte für jeden Peak; diese Werte sind in Tabelle $V$ aufgeführt. Nun wollen wir die jeweiligen $\mathrm{C}_{4}$-Alkohole den einzelnen Peaks zuordnen. Dazu benutzen wir die Inkremental-Werte, wie sie von Wehrli und Kováts [6] für dieses betreffende Paar flüssiger Phasen publiziert worden sind; sie sind zum Teil in Tabelle VI abgedruckt; und hier beschäftigen uns die Werte, die den Alkoholen entsprechen.

Unter Verwendung der in Tabelle VI angegebenen Werte können wir den erwarteten $\Delta \mathrm{I}$-Wert für jeden der vier Butylalkohole berechnen. Der Basiswert ist immer 453, und die abzuziehenden Inkremental-Werte setzen sich ge-

Tabelle VI. Retentionsindex-Inkremente für die Berechnung von $\Delta I$

Flüssige Phasen: $\quad$ polar - Emulphor $O$ unpolar - Apiezon $L$

\begin{tabular}{|c|c|c|c|c|c|c|c|c|c|c|}
\hline \multirow{3}{*}{ Typ } & \multirow{3}{*}{$\mathrm{X}$} & \multirow{3}{*}{ Klasse } & \multirow{3}{*}{$\begin{array}{l}\text { Basis- } \\
\text { wert }\end{array}$} & \multicolumn{4}{|c|}{$\mathbf{R}=$} & \multirow{2}{*}{\multicolumn{3}{|c|}{$\begin{array}{c}\text { Methylgruppe } \\
\text { an R } \\
\text { in Stellung }\end{array}$}} \\
\hline & & & & \multirow{2}{*}{$C_{1}$} & \multirow{2}{*}{$C_{2}$} & \multirow{2}{*}{$C_{3}$} & \multirow{2}{*}{$\begin{array}{c}\mathrm{C}_{4} \\
\text { und } \\
\text { höher }\end{array}$} & & & \\
\hline & & & & & & & & $\alpha$ & $\beta$ & $\gamma$ \\
\hline $\mathbf{R}_{1}$ & $\mathrm{OH}$ & Alkohol & 453 & -57 & -76 & -80 & -82 & -19 & -4 & -2 \\
\hline $\mathrm{R}_{2}-\mathrm{C}-\mathrm{X}$ & $\mathrm{Cl}$ & Chlorid & 174 & -21 & -33 & -39 & -44 & -12 & -6 & -5 \\
\hline${\stackrel{1}{\mathbf{R}_{3}}}^{\mid}$ & $\mathrm{Br}$ & Bromid & 184 & -21 & -33 & -39 & -44 & -12 & -6 & -5 \\
\hline
\end{tabular}


mäß der nachstehenden Aufstellung für die vier Fälle zusammen:<smiles>CCCCO</smiles>

$$
\begin{aligned}
& \Delta \mathrm{I}=453-80=373 \\
& \begin{array}{l}
\mathrm{R}_{1}=\mathrm{C}_{3} \\
\mathrm{R}_{2}=\mathrm{H} \\
\mathrm{R}_{3}=\mathrm{H}
\end{array}
\end{aligned}
$$

Butanol-2<smiles>CCC(C)O</smiles>

$\Delta \mathrm{I}=453-133=320$

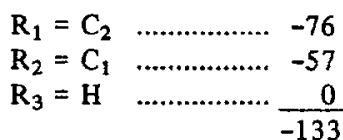

2-Methyl-propanol-1<smiles>CC(C)CO</smiles>

2-Methyl-propanol-2<smiles>CC(C)(C)O</smiles>

$$
\begin{aligned}
& \Delta I=453-171=282 \\
& \mathrm{R}_{1}=\mathrm{C}_{1} \quad \ldots \ldots \ldots \ldots \ldots \ldots \ldots \ldots \\
& \mathbf{R}_{2}=C_{1} \quad \ldots \ldots \ldots \ldots \ldots \ldots . . .57 \\
& \mathrm{R}_{3}=\mathrm{C}_{1} \ldots \ldots \ldots \ldots \ldots \ldots \ldots \ldots \ldots-\frac{-57}{-171}
\end{aligned}
$$

Auf der rechten Seite der Tabelle V sind die berechneten Werte mit den gemessenen verglichen und daraufhin die Peaks den einzelnen Komponenten zugeordnet. Wie man sieht, ist die Übereinstimmung gut. Natürlich können wir keine vollständige Übereinstimmung zwischen den berechneten und den gemessenen Werten erwarten; eine Utbereinstimmung innerhalb weniger Indexeinheiten ist durchaus zufriedenstellend.

Diese Berechnung zeigt auch, daß nicht nur die funktionelle Gruppe selbst, sondern auch ihre Stellung wichtig ist. In anderen Worten, man kann nicht einfach einen Inkremental-Wert für die Hydroxyl-Gruppe angeben, sondern man muß auch die „Wechselwirkung" zwischen der aktiven Gruppe und den anderen im Molekül vorhandenen Gruppen berücksichtigen.

Lassen Sie uns ein weiteres Beispiel aus der Publikation von Wehrli und Kováts [6] anfuhren. Bei der Untersuchung von ätherischen ölen wurde ein Terpenalkohol gefunden, aber seine genaue Struktur war unsicher. Nachstehend sind die vier möglichen Strukturen gezeigt, zusammen mit den berechneten $\Delta \mathrm{I}$-Werten, die diesen Strukturen entsprechen:<smiles>CC(C)(O)C1CC=C(I)CC1</smiles><smiles>CC(C)C1(O)CC=CCC1</smiles><smiles>CC1CCC([Al])=CC1O</smiles><smiles>CC(C)C1CCC(O)(O)CC1</smiles>

$\Delta \mathrm{I}=281$

$\Delta \mathrm{I}=333$

$\Delta \mathrm{I}=304$
Durch Berechnung des Retentionsindex des betreffenden Peaks auf den beiden Säulen mit den flüssigen Phasen Emulphor $\mathrm{O}$ bzw. Apiezon L wurde ein Wert von 270 für $\Delta \mathrm{I}$ ermittelt. Wie man sieht, kommt der berechnete $\Delta \mathrm{I}$ Wert für die Struktur II dem gemessenen Wert am nächsten, und deshalb wurde diese Struktur als die richtige angenommen. Nachfolgende Untersuchungen mit GC-MS bestätigten diesen Befund.

\section{Rohrschneider-Konstanten}

Auf der Grundlage der Arbeiten von Kováts schlug Rohr. schneider 1965-1966 vor, beim Begriff der Bedeutung und Verwendung des Retentionsindex und des Inkrements noch einen Schritt weiterzugehen [36, 37]. Bei der Untersuchung des Retentionsverhaltens auf verschiedenen stationären Phasen fand er, daß die Retentionsindexdifferenz $\Delta \mathrm{I}$ sich aus mehreren Beiträgen zusammensetzt, die jeweils durch zwei Faktoren bestimmt werden: solchen, die spezifisch für das Gelöste (die Proben-Komponente) sind und sich bei Änderung der flüssigen Phase nicht mit ändern, und solchen, die für die flüssige Phase spezifisch sind und nicht von dem jeweiligen Gelösten abhängen, aber mit einer begrenzten Anzahl ausgewählter Testsubstanzen in Beziehung stehen. Die ersteren werden durch die Symbole $a, b, c$, d und e charakterisiert und die letzteren durch die Symbole $x, y, z, u$ und $s$. ${ }^{*}$ Definitionsgemäß wird das Retentionsindex-Inkrement $\Delta I$ eines Gelösten auf einer gegebenen stationären Phase ausgedrückt als

$$
\Delta \mathrm{I}=\mathrm{ax}+\mathrm{by}+\mathrm{cz}+\mathrm{du}+\mathrm{es}
$$

Betrachten wir einmal die Bedeutung dieser Konstanten.

Phasenspezifische Konstanten. Man erhält sie, indem man fünf Testsubstanzen auswählt und für jede den Wert $\Delta \mathrm{I} /$ 100 bestimmt. Für Benzol, die erste Testsubstanz, ist das zum Beispiel

$$
\mathrm{x}=\frac{\Delta \mathrm{I}(\text { Benzol })}{100}=\frac{\mathrm{I}^{\text {Phase }}(\text { Benzol })-\mathrm{I}^{\text {Squalan }}(\text { Benzol })}{100}
$$

Die anderen von Rohrschneider vorgeschlagenen Testsubstanzen sind A thanol, Methyläthylketon, Nitromethan und Pyridin, und die jeweiligen Symbole für die $\Delta I / 100$ Werte sind $y, z, u$ und $s$. Jede Testsubstanz stellt die Wechselwirkungen für die betreffende flüssige Phase für eine bestimmte (in der Test-Probe enthaltene) Gruppe dar, und wir werden uns mit dieser Frage später im Teil III dieser Serie befassen.

Probenspezifische Konstanten. Wie schon erwähnt, beziehen diese sich nur auf das jeweilige Gelöste (Probenkomponente) und sind unabhängig von der fluissigen Phase. Sie können ermittelt werden, indem man die phasenspezi-

\footnotetext{
* In seiner ersten Publikation [36] hielt Rohrschneider drei Testsubstanzen für ausreichend, und er benutzte die Symbole $x, y$ und $z$ zur Kennzeichnung der drei entsprechenden phasenspezifischen Konstanten, dic sich auf diese drei Testsubstanzen bezogen. Als er cin Jahr später zwei weitere Testsubstanzen den drei anderen schon festgelegten hinzufügte [37], wollte er die Symbole für dic schon vorhandenen Konstanten nicht ändern. Dies ist der Grund für die unregelmäßige Reihenfolge der Symbole.
} 
fischen Konstanten für mindestens fünf flüssige Phasen bestimmt und dann den $\Delta \mathrm{I}$-Wert für das betreffende Gelöste auf den fünf (oder mehr) Phasen relativ zu Squalan mißt; schließlich sind die Gleichungen für die Konstanten a ...e zu lösen:

$$
\begin{aligned}
& I^{\text {Ph.1 }}-I^{\text {Squalan }}=\mathrm{ax}_{1}+\mathrm{by}_{1}+\mathrm{cz}_{1}+\mathrm{du}_{1}+\mathrm{es}_{1} \\
& \mathrm{I}^{\mathrm{Ph} .2}-\mathrm{I}^{\text {Squalan }}=\mathrm{ax}_{2}+\mathrm{by}_{2}+\mathrm{cz}_{2}+\mathrm{du}_{2}+\mathrm{es}_{2} \\
& \vdots \\
& \mathrm{I}^{\text {Ph.5 }}-\mathrm{I}^{\text {Squalan }}=\mathrm{ax}_{5}+\mathrm{by}_{5}+\mathrm{cz}_{5}+\mathrm{du}_{5}+\mathrm{es}_{5}
\end{aligned}
$$

wobei die Indexziffern 1 ...5 bei den phasenspezifischen Konstanten sich auf die fünf Phasen beziehen. Für eine eingehende mathematische Abhandlung siehe die Publikation von Rohrschneider [37].

Wir haben gesehen, daß die phasenspezifischen Konstanten $\mathrm{x} \ldots \mathrm{s} \Delta \mathrm{I} / 100$-Werte für die spezifische Testsubstanz auf dieser Phase darstellen. Deshalb ist leicht einzusehen, daß die Konstanten a ... e der fünf Testsubstanzen, die zur Bestimmung der phasenspezifischen Konstanten verwendet werden, definitionsgemäße Konstanten sind. Diese sind in Tabelle VII aufgeführt.

Tabelle VII. Probenspezifische Rohrschneider-Konstanten für die fünf Testsubstanzen

\begin{tabular}{lcc|c|c|c}
\hline & a & b & c & d & e \\
\hline Benzol & 100 & 0 & 0 & 0 & 0 \\
Äthanol & 0 & 100 & 0 & 0 & 0 \\
Methyläthylketon & 0 & 0 & 100 & 0 & 0 \\
Nitromethan & 0 & 0 & 0 & 100 & 0 \\
Pyridin & 0 & 0 & 0 & 0 & 100 \\
\hline
\end{tabular}

Obwohl sowohl die phasenspezifischen als auch die probenspezifischen Konstanten von Rohrschneider vorgeschlagen worden sind, bezeichnet man in der Praxis mit dem Ausdruck „Rohrschneider-Konstanten" nur die phasenspezifischen Konstanten. Der Grund dafür liegt darin, daß die Anwendung des Rohrschneider'schen Konzepts zur Charakterisierung von Gelösten und die Vorhersage des $\Delta \mathrm{I}$-Wertes für ein gegebenes Gelöstes auf einer gegebenen Phase ziemlich kompliziert ist und Daten erfordert, die nicht unmittelbar zur Verfügung stehen; andererseits hat sich die Verwendung der phasenspezifischen Konstanten zur Charakterisierung einer flüssigen Phase immer mehr durchgesetzt, im wesentlichen dank der Arbeiten von Supina und Rose [38] und McReynolds [18]. Das wird Gegenstand von Teil III dieser Serie sein.

\section{Zusammenhang zwischen Retentionsindex und relativer Retention}

Die Gas-Chromatographie ist ein Trennverfahren; wenn wir also ein gegebenes Gemisch analysieren wollen, ist unsere erste Frage, ob die Komponenten $A$ und B unter bestimmten Versuchsbedingungen (flüssige Phase, Tempe- ratur) auf einer gegebenen Säule getrennt werden können. Wir kennen bereits (Teil I, Gl. 6) die grundsätzliche Gleichung, in der Peak-Auflösung (R), relative Retention ( $\alpha$ ) und Verteilungs- oder Kapazitäts-Faktor (k) mit der Trenn. leistung der Säule (Anzahl der theoretischen Böden, n) in Beziehung gesetzt werden:

$$
\mathrm{n}=16 \mathrm{R}^{2}\left(\frac{\alpha}{\alpha-1}\right)^{2}\left(\frac{\mathrm{k}_{2}+1}{\mathrm{k}_{2}}\right)^{2}
$$

Aus dieser Gleichung ergibt sich die Auflösung $\mathrm{R}$ als:

$$
\mathrm{R}=\frac{\sqrt{\mathrm{n}}}{4}\left(\frac{\alpha-1}{\alpha}\right)\left(\frac{\mathrm{k}_{2}}{\mathrm{k}_{2}+1}\right)
$$

Aus der allgemeinen Kenntnis der GC wird man oft gefühlsmäßig wissen, ob gegebene relative Retentionswerte leichte oder schwierige Trennprobleme darstellen. Zum Beispiel werden sich zwei Peaks mit einem Wert $\alpha>1,10$ im allgemeinen mit einer guten gepackten Säule trennen lassen, während bei $\alpha<1,05$ die Trennung selbst auf langen Kapillarsäulen schwierig ist. Für die Auswahl der Analysenbedingungen sollte man schon annähernd wissen, wel. ches die zu erwartende relative Retention für das am schwierigsten zu trennende Peak-Paar sein wird. Hierbei stellt sich die Frage: kann man diesen Wert aus dem Retentionsindex ermitteln?

Wir wollen diese Frage kurz diskutieren; Einzelheiten siehe [39].

Wenn wir zwei benachbarte Peaks nehmen, die den Verbindungen $n$ und $m$ entsprechen - wobei $t_{R(m)}^{\prime}>t_{R(n)}^{\prime}$ und also $I_{m}>I_{n}$ ist - und die innerhalb der Peaks der gleichen beiden n-Paraffine auftreten, dann können wir die Differenz der beiden Retentionsindices i ausdrücken als:

$$
\begin{aligned}
i=I_{m}-I_{n} & =100 \frac{\log t_{R(m)}^{\prime}-\log t_{R(n)}^{\prime}}{\log t_{R(z+1)}^{\prime}-\log t_{R(z)}^{\prime}}= \\
& =100 \frac{\log \alpha_{m / n}}{\log r_{(z+1) / z}}
\end{aligned}
$$

wobei

$$
\begin{aligned}
\alpha_{m / n} & =t_{R(m)}^{\prime} / t_{R(n)}^{\prime} \\
r_{(z+1) / z} & =t_{R(z+1)}^{\prime} / t_{R(z)}^{\prime}
\end{aligned}
$$

Wie schon in Teil I erläutert, stellen beide Symbole, $\alpha$ und $\mathrm{r}$, relative Retentionen dar, mit dem einzigen Unterschied, daß $\alpha$ immer die relative Retention zweier aufeinanderfolgender Peaks ausdrückt.

Wir haben schon in Teil I gesehen, daß bei einer gegebenen Temperatur und flüssigen Phase $r_{(z+1) / z}$ konstant und unabhängig von $z$ ist. Daher ist $100 / \log r_{(z+1) / z}$ ebenfalls eine Konstante, und wir können Gl. 25 wie folgt ausdrücken:

$$
\mathrm{i}=(\text { konstant }) \log \alpha_{\mathrm{m} / \mathrm{n}}
$$

oder

$$
\log \alpha_{m / n}=(\text { konstant }) \mathrm{i}
$$


Man kann leicht die relative Retention zweier aufeinanderfolgender $n$-Paraffine bei jeder beliebigen Temperatur auf jeder interessierenden Phase bestimmen und daraus Tabellen oder Diagramme aufstellen; wenn man den betreffenden Wert vorliegen hat, dann kann man aus dem Unterschied der beiden Retentionsindices die relative Retention zweier zu trennender benachbarter Peaks berechnen, und wenn einige Angaben über die Trennleistung der Säule vorliegen, dann läßt sich auch berechnen, ob eine gegebene Säule für die Trennung ausreicht.

Gl. 28b kann noch weiter vereinfacht werden. Es ist eine bekannte mathematische Regel, daß für kleine Werte von $\chi \log (1+\chi) \simeq \chi$ ist. Anders gesagt:

$$
\begin{aligned}
& \chi \simeq(\text { konstant }) \mathrm{i} \\
& \chi=\frac{\alpha-1}{100}
\end{aligned}
$$

Das bedeutet, daß eine Anderung von z. B. $\alpha=1,05$ auf $\alpha=1,10$ eine Anderung um etwa $100 \%$ im Wert von $\mathrm{i}$ bewirkt. Zum Beispiel sind die betreffenden Werte auf Squalan bei $100^{\circ} \mathrm{C}$ :

$$
\begin{array}{ll}
\alpha=1,05 & \mathrm{i}=6,5 \\
\alpha=1,10 & \mathrm{i}=12,5
\end{array}
$$

Da die Retentionsindex-Werte üblicherweise auf die nächste ganze Zahl auf- oder abgerundet werden, wird die Berechnung von i nur annähernde Werte ergeben. Jedoch liegen sie den tatsächlichen Werten ziemlich nahe, und da solche Berechnungen nur vorgenommen werden, um die geeigneten Analysenbedingungen zu ermitteln, haben die auftretenden Abweichungen keine ernsthafte Auswirkung.

Das folgende Beispiel illustriert die Verwendung von $i$ für eine praktische Berechnung [39].

Wir möchten wissen, welche Auflösung wir von einer Kapillarsäule von $150 \mathrm{ft}$. Länge mit der flüssigen Phase Squalan bei $45^{\circ} \mathrm{C}$ für das Peak-Paar 2,5-Dimethylhexan und 2,4-Dimethylhexan erwarten können. Bei dieser Temperatur hat unsere Säule eine theoretische Bodenhöhe (HETP) von $0,77 \mathrm{~mm}$ und einen Verteilungsfaktor $(\mathrm{k})$ von 9,42 für 2,4-DMHx, und die entsprechenden RetentionsindexWerte sind:

\section{2,5-Dimethylhexan 729}

2,4-Dimethylhexan 732,5.

Daher ist $\mathbf{i}=732,5-729=3,5$.

Wir haben früher ermittelt, daß bei $45^{\circ} \mathrm{C} r_{(z+1) / z}=2,745$. Daher können wir aus G1. $25 \alpha$ berechnen:

$$
\log \alpha=\frac{(3,5)(\log 2,745)}{100}=0,01314
$$

und also ist $\alpha=1,036$.
Die Beziehung zwischen HETP(h), Säulenlänge (L) und Anzahl der theoretischen Böden ( $n$ ) ist bereits in Gl. 3 ausgedrückt:

$$
\mathrm{n}=\frac{\mathrm{L}}{\mathrm{h}}
$$

Da $\mathrm{h}=0,77 \mathrm{~mm}$ und $\mathrm{L}=150 \mathrm{ft}=45720 \mathrm{~mm}$, ist $\mathrm{n}=$ 59400 . Wenn man die betreffenden Werte in Gl. 6a einsetzt, erhält man eine Auflösung von $R=1,91$.

Wir haben diese Berechnung überprüft, indem wir die Probe auf der Säule analysierten, und erhielten $\alpha=1,034$ und $R=1,84$. Diese Ubereinstimmung ist sehr gut.

\section{Literatur}

[23] J. K. Haken, J. Chromatog. Sci. 11, 144-150 (1973).

[24] L. Rohrschneider, J. Chromatog. Sci. 11, 160-166 (1973).

[25] R. A. Keller, J. Chromatog. Sci. 11, 188-190 (1973).

[26] J. M. Takacs, J. Chromatog. Sci. 11, 210-215 (1973).

[27] $P$. Chovin and $J$. Lebbe, in "Journées Internationales d'Etude des Méthodes de Séparation Immédiate et de Chromatographie", ed. J. Tranchant, G.A.M.S., Paris, 1961; pp. 90-103.

[28] P. Chovin and J. Lebbe, J. Gas Chromatog. 4, 37-41 (1966).

[29] J. Takács, M. Rockenbauer and I. Olácsi, J. Chromatog. 42, 19-28 (1969).

[30] E. B. Molnár, P. Moritz and J. Takács, J. Chromatog. 66, 205-212 (1972).

[31] Data Subcommittee of the GC Discussion Group, J. Gas Chromatog. 4, 1-3 (1966).

[32] J. Takács, C. Szita and G. Tarián, J. Chromatog. 56, 1-12 (1971).

[33] J. Takács, Zs. Tálas, I. Bernát, Gy. Czakó and A. Fischer, J. Chromatog. 67, 203-212 (1972).

[34] J. M. Takács, E. Kocsi, E. Garamvölgyi, E. Eckhart, T. Lombosi, Sz. Nyiredy Jr., I. Borbély and Gy. Krasznai, J. Chromatog. 81, 1-8 (1973).

[35] G. Costello, M. Lunardelli and M. Berg, J. Chromatog. 76, 31-44 (1973).

[36] L. Rohrschneider, J. Chromatog. 17, 1-12 (1965).

[37] L. Rohrschneider, J. Chromatog. 22, 6-22 (1966).

[38] W. R. Supina and L. R. Rose, J. Chromatog. Sci. 8, 214-217 (1970).

[39] L. S, Ettre and K, Billeb, J. Chromatog. 30, 12-16 (1967). 


\title{
Le système d'indice de rétention; son utilisation pour l'identification des substances et la caractérisation des phases fixes liquides*
}

\section{2eme partie: Corrélation entre indice de rétention, structure et caractéristiques analytiques**}

\author{
L. S. Ettre \\ The Perkin-Elmer Corporation, Norwalk, Connecticut 06856, U.S.A.
}

Le premier article de cette série*) traitait de la signification de l'indice de rétention et du moyen de le calculer. Sa valeur ne dépendant pas des caractéristiques de l'appareillage mais seulement de la phase fixe (liquide) et de la température, il est évident que l'indice de rétention convient parfaitement pour l'identification des substances.

Cependant la notion d'indice de rétention offre bien d'autres possibilités que la simple identification des solutés à l'aide de tables ("table matching" selon l'expression de Schomburg et Dielmann [16]), méthode qui consiste à comparer les valeurs d'indice de rétention mesurées à des valeurs de référence collationnées dans des tables. En effet, les valeurs d'indice de rétention peuvent aussi être mises en relation avec la structure des substances analysées (solutés), la rétention relative à deux substances et les variations des caractéristiques de rétention en fonction des conditions d'analyse (en particulier, la température). Ces relations peuvent être exprimées comme des fonctions de divers "incréments" de l'indice de rétention; ainsi dans ce second article de la série, nous exposerons brièvement la signification des divers "incréments" de l'indice de rétention.

Il faut remarquer que les publications concernant ces relations, en particulier les relations existant entre les "incréments" d'indice de rétention et la structure, sont très nombreuses et nous ne pouvons ni entrer dans les détails, ni même donner une bibliographie complète. Les lecteurs intéressés par une information plus complète pourront par exemple consulter la collection d'articles récemment publiés sur ce sujet $[16,23-26]$ qui donne aussi une bibliographie détaillée.

Notre exposé sera centré autour de cinq types de différences d'indice de rétention ou, selon l'expression habi-

*) Le premier article de cette série a été publié dans le numéro de décembre 1973 de Chromatographia.

**) Références, équations, tables et figures font l'objet d'une numérotation unique pour toute la série d'articles. Pour les références 1 à 22 , voir la première partie. tuelle, cinq types "d'incréments" d'indice. Leur définition est donnée ci-dessous ainsi que leur symbole quand il en existe un.

La différence des indices de rétention d'un soluté donné mesurés, avec la même phase fixe liquide, à deux températures différentes ...............

$\mathrm{La}$ différence des indices de rétention de deux solutés appartenant à la même série d'homologues et différant seulement d'un atome de carbone, mesurés avec la même phase fixe liquide, à la même température.

La différence des indices de rétention de deux solutés l'un étant le corps étudié, l'autre l'hydrocarbure saturé à chaîne droite ayant le même nombre d'atomes de carbone, mesurés avec une phase fixe liquide non polaire, à la même température $\ldots \ldots \ldots \ldots \ldots \ldots \ldots \mathrm{H}^{\mathrm{NP}}$ La différence des indices de rétention d'un soluté donné mesurés dans deux phases liquides différentes, l'une polaire et l'autre non polaire, à la même température ....................... $\Delta \mathrm{I}$

La différence des indices de rétention de deux solutés différents dans la même phase liquide, à la même température $\ldots \ldots \ldots \ldots \ldots \ldots \ldots \ldots \ldots$ i

La première valeur - $\partial \mathrm{I}$ - traduit l'influence de la température sur l'indice de rétention; les trois valeurs suivantes $-\mathrm{H}^{\mathrm{NP}}, \Delta \mathrm{I}, \mathrm{i}$ - traduisent le lien existant entre l'indice de rétention et la structure moléculaire du soluté, tandis que la dernière valeur - $\mathbf{i}$ - permet d'apprécier le degré de séparation de deux substances sur une colonne donnée.

\section{Influence de la température sur l'indice de rétention}

Nous avons déjà mentionné dans la première partie que les valeurs de rétention relative dépendent de la température. Cette dépendance peut être exprimée par l'équation suivante $[27,28]$ :

$$
\log r=a+\frac{b}{T}
$$

dans laquelle $T$ est la température en degrés Kelvin et a et $\mathrm{b}$ des constantes. 
Nous avons vu dans l'équation 12 (première partie) que l'indice de rétention peut être exprimé comme une fonction de deux valeurs de rétention relative:

$$
I=100 z+100 \frac{\log r_{x / z}}{\log r_{(z+1) / z}}
$$

Naturellement, les valeurs des deux rétentions relatives dépendent de la température et peuvent être exprimées par l'équation 14; seules les constantes diffèrent:

$$
\begin{aligned}
& \log r_{x / z}=a+\frac{b}{T} \\
& \log r_{(z+1) / z}=c+\frac{d}{T}
\end{aligned}
$$

En introduisant ces équations dans l'équation 12, nous pouvons écrire que I est proportionnel à $(\mathrm{aT}+\mathrm{b}) /(\mathrm{cT}+\mathrm{d})$ :

$$
I \propto \frac{a T+b}{c T+d}
$$

\section{$a, b, c$ et d étant des constantes.}

Des études détaillées ont été publiées (voir par exemple $[29,30])$ et ont montré que la relation réelle entre l'indice de rétention et la température de la colonne peut généralement être traduite par une équation d'Antoine:

$$
I=A+\frac{B}{T+C}
$$

dans laquelle $\mathrm{A}, \mathrm{B}$ et $\mathrm{C}$ sont des constantes dépendant du soluté et de la phase fixe liquide. L'équation 17 correspond à une hyperbole; cependant, il a été démontré $[12,28]$ que dans l'intervalle de température utilisé, en pratique, la courbe représentant les variations de I en fonction de $\mathrm{T}$, présente une portion linéaire et nous pouvons par conséquent écrire:

$$
\mathrm{I} \simeq \mathrm{a}^{\prime} \mathrm{T}+\mathrm{b}^{\prime}
$$

$a^{\prime}$ et $b^{\prime}$ étani des constantes. Cette relation linéaire couvre généralement une gamme de température plus étendue pour une phase fixe liquide non polaire que pour une phase polaire.

Dans la plupart des cas, on a $a^{\prime}>0$ et ainsi, l'indice de rétention croît quand la température augmente; il existe cependant des cas où c'est l'inverse.

En raison de la relation approximativement linéaire que traduit l'équation 18 , on peut exprimer l'influence de la température sur l'indice de rétention en donnant l'incrément pour une différence de température donnée, habituellement $10^{\circ} \mathrm{C}$, et utiliser alors cette valeur pour calculer l'indice de rétention à une température différente de celle à laquelle il a été déterminé. Par exemple, si les valeurs suivantes [31] sont données pour l'acétate de n-propyle:

$$
\mathrm{I}_{80}^{\text {Carbowax }}{ }^{\circ} \mathrm{C00}=1043 \quad \partial \mathrm{I} / 10^{\circ}=4,25
$$

on peut calculer par exemple que la valeur à $60^{\circ} \mathrm{C}$ est $1043-(2 \times 4,25)=1034,5$. La valeur réelle est 1035 .
On doit faire ici deux remarques. La première est que l'on ne doit pas trop intrapoler ou extrapoler parce que la relation entre I et $\mathrm{T}$ cesse d'être linéaire pour une large gamme de température et que l'on doit être particulièrement prudent lorsque l'on a affaire à des phases polaires. La seconde est qu'il vaut mieux calculer les valeurs de $\partial \mathrm{I} / \partial \mathrm{T}$ à partir d'une courbe pour l'établissement de laquelle on a pu éviter les inexactitudes de mesure.

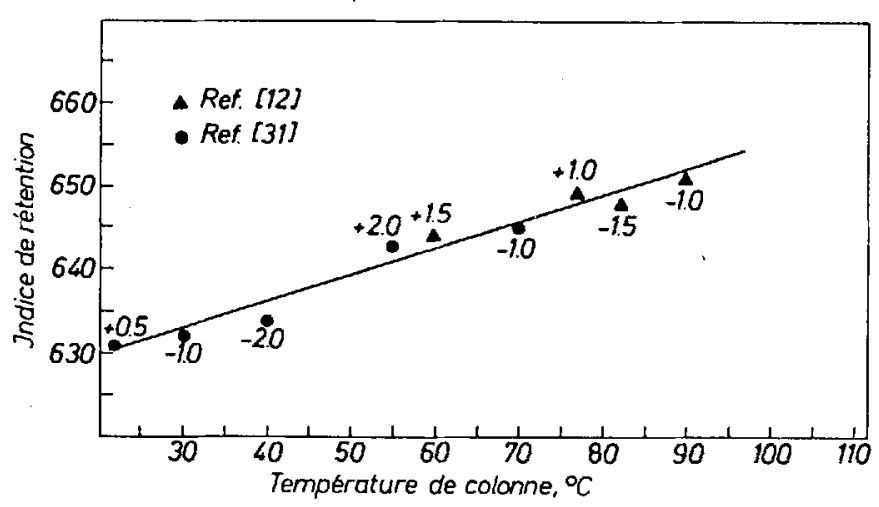

Fig. 4

- Valeurs d'indice de rétention du benzène sur squalane en fonction de la température. Les nombres inscrits près des points indiquent la déviation (en unités d'indice) de la valeur par rapport à la droite.

Par exemple, la figure 4 montre la courbe des valeurs d'indice de rétention du benzène sur squalane provenant de deux sources et indique aussi les écarts entre la courbe et les mesures isolées [12]. La courbe représente la meilleure concordance entre ces valeurs et nous pourrions ainsi calculer la valeur de $\partial \mathrm{I} / \partial \mathrm{T}$ à partir de la courbe. Par exemple, nous pouvons lire les valeurs suivantes:

$$
I_{30^{\circ}}=633 \quad I_{60^{\circ}}=643 \quad I_{90^{\circ}}=652
$$

La différence pour un intervalle de $60^{\circ} \mathrm{C}$ est $652-633$ $=19$ unités d'indice et par conséquent $\partial \mathrm{I} / 10^{\circ} \mathrm{C}=3,2$.

Tableau IV. Valeurs d'indice de rétention du benzène sur squalane

\begin{tabular}{c|cccc}
\hline $\begin{array}{c}\text { Température } \\
{ }^{\circ} \mathrm{C}\end{array}$ & $\begin{array}{l}\text { Données de la } \\
\text { littérature }^{\mathrm{a}}\end{array}$ & $\begin{array}{l}\text { Valeurs } \\
\text { calculées }^{\mathrm{b}}\end{array}$ & Différence \\
\hline 22 & 631 & 631 & 0 \\
30 & 632 & 633 & +1 \\
40 & 634 & 637 & +3 \\
55 & 643 & 641 & -2 \\
60 & $644^{*}$ & 643 & -1 \\
70 & 645 & 646 & +1 \\
77 & $649^{*}$ & 648 & -1 \\
82 & $648^{*}$ & 650 & +2 \\
90 & $651^{*}$ & 653 & +2 \\
\hline
\end{tabular}

a les valeurs marquées d'un astérisque sont tirées de la référence 12 , les autres proviennent de la référence 31

b en utilisant $\mathrm{I}_{60^{\circ}}=643$ et $\partial \mathrm{r} / 10^{\circ}=3,2$ 
Dans une liste des indices de rétention, nous devrions ainsi donner pour le benzène sur squalane les valeurs:

$$
\mathrm{I}_{60}^{\text {squalane }}=643 \quad \partial \mathrm{C} / 10^{\circ} \mathrm{C}=3,2
$$

et calculer l'indice de rétention aux autres températures, à partir de ces valeurs. Dans le tableau IV, on indique les valeurs mesurées ayant servi à établir la courbe de la figure 4 et les valeurs calculées de cette manière. On voit que la concordance est assez bonne, à 3 unités d'indice près, ce qui est à peu près actuellement la précision que l'on peut attendre en utilisant des données provenant de laboratoires différents.

Naturellement les courbes de I en fonction de $\mathrm{T}$ peuvent aussi être utilisées pour traduire l'influence de la température sur l'indice de rétention; les figures 5 et 6 représentent de telles courbes correspondant à un certain nombre d'hydrocarbures sur squalane comme phase fixe liquide [12]. Ces courbes permettent de lire directement la valeur de l'indice de rétention d'une substance donnée à n'importe quelle température et présentent en outre une double utilité: elles permettent de déterminer rapidement la température optimale de séparation des substances d'un mélange donné et fournissent des renseignements complémentaires pour l'identification qualitative de certains types de composés sur le chromatogramme donné par un mélange complexe.

La possibilité d'évaluer la température qui convient à une analyse sera mieux illustrée par un exemple. Supposons que nous ayons un mélange renfermant les trois produits suivants:

méthylcyclohexane

2,4-diméthylhexane

2,5-dimethylhexane

Il ressort de la figure 6 qu'on ne devrait analyser ce mélange ni aux environs de $60^{\circ} \mathrm{C}$, ni à $85^{\circ} \mathrm{C}$ parce qu'à ces températures le méthylcyclohexane sortira de la colonne en même temps que l'un des deux carbures

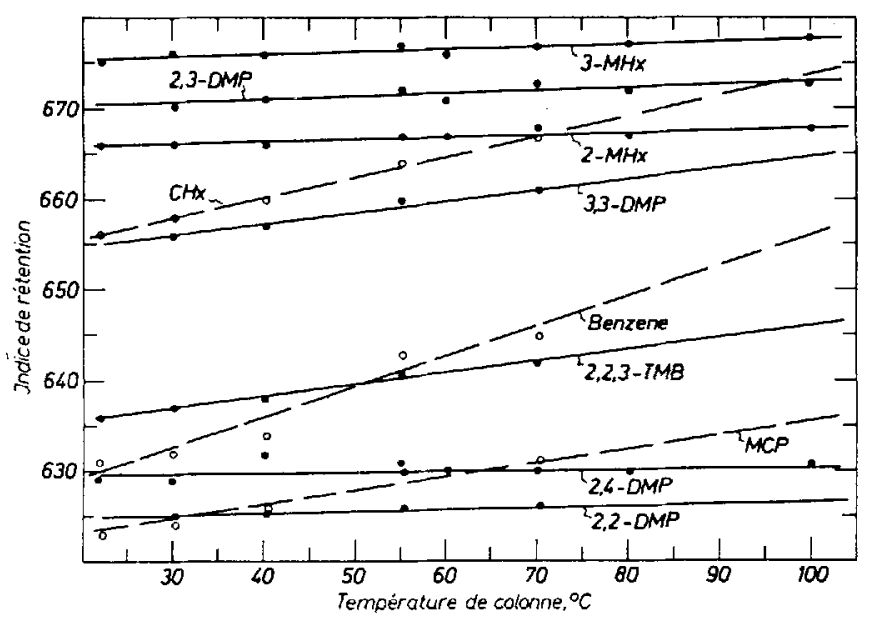

Fig. 5

- Valeurs des indices de rétention des hydrocarbures sur squalane en fonction de la température. Domaine de valeurs d'indice: $620-680$.

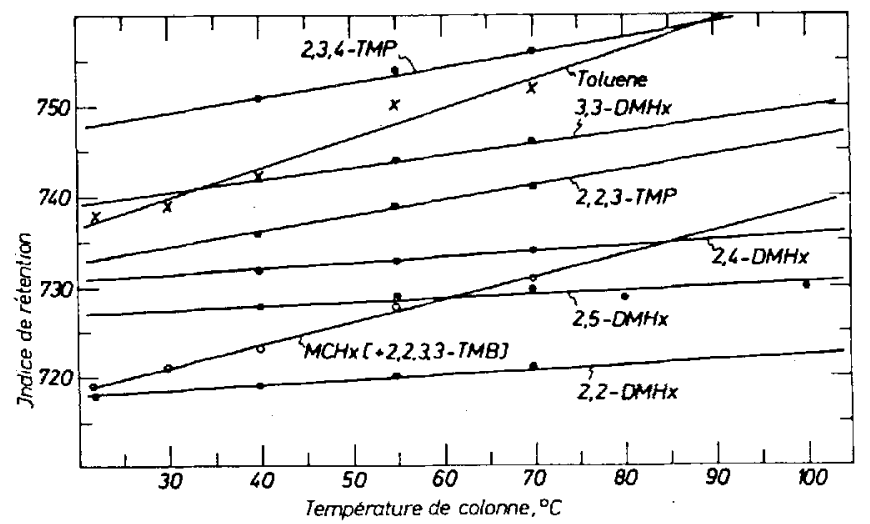

Fig. 6

- Valeurs des indices de rétention des hydrocarbures sur squalane en fonction de la température. Domaine de valeurs d'indice: 710-760. Abréviations utilisées dans les figures 5 à 6:

TMB = triméthylbutane

DMP $=$ diméthylpentane

TMP = trimëthylpentane

$\mathrm{MHx}=$ méthythexane

$\mathrm{DMHx}=$ diméthylhexane

$\mathrm{MCP}=$ méthylcyclopentane

$\mathrm{CHx}=$ cyclohexane

$\mathrm{MCHx}=$ méthylcyclohexane

aliphatiques saturés isomères. On devra donc choisir une température d'environ $40-50^{\circ} \mathrm{C}, 75^{\circ} \mathrm{C}$ ou $100^{\circ} \mathrm{C}$.

La possibilité d'utiliser de telles courbes comme source complémentaire de renseignements pour l'identification qualitative repose sur les différences de pente des courbes. Par exemple, nous pouvons voir que sur squalane les courbes des carbures aliphatiques saturés isomères ont une pente d'autant plus grande qu'ils ont d'avantage de chaînes latérales; comparer le 3-méthylhexane au 3,3diméthylpentane ou le 2,4-diméthylhexane au 2,2,3triméthylpentane. Les courbes des hydrocarbures cycliques et des hydrocarbures aromatiques (et des hydrocarbures aliphatiques isomères saturés très ramifiés) ont une pente si grande que ces courbes coupent réellement les courbes des hydrocarbures aliphatiques saturés isomères à chaîne branchée; il en résulte que, dans un mélange complexe, les pics des hydrocarbures cycliques et des hydrocarbures aromatiques pourraient occuper une position différente par rapport aux pics des hydrocarbures aliphatiques isomères à chaîne ramifiée (et naturellement des hydrocarbures à chaine droite) si l'analyse est effectuée à des températures différentes. A titre d'exemple, reprenons l'exemple précédent d'un mélange temaire renfermant du méthylcyclohexane (MCHx), du 2,4- et du 2,5diméthylhexane (DMHx). Il est facile de déduire de la figure 6 , que la succession des pics dépend de la température d'analyse:

en-dessous de $60^{\circ} \mathrm{C}: \quad \mathrm{MCHx} \quad-2,5$-DMHx $-2,4$-DMH à $60-85^{\circ} \mathrm{C}$ : au-dessus de $85^{\circ} \mathrm{C}$ :
2,5-DMHx - MCHx -2,4-DMH 2,5-DMHx - 2,4-DMHx - MCHx 

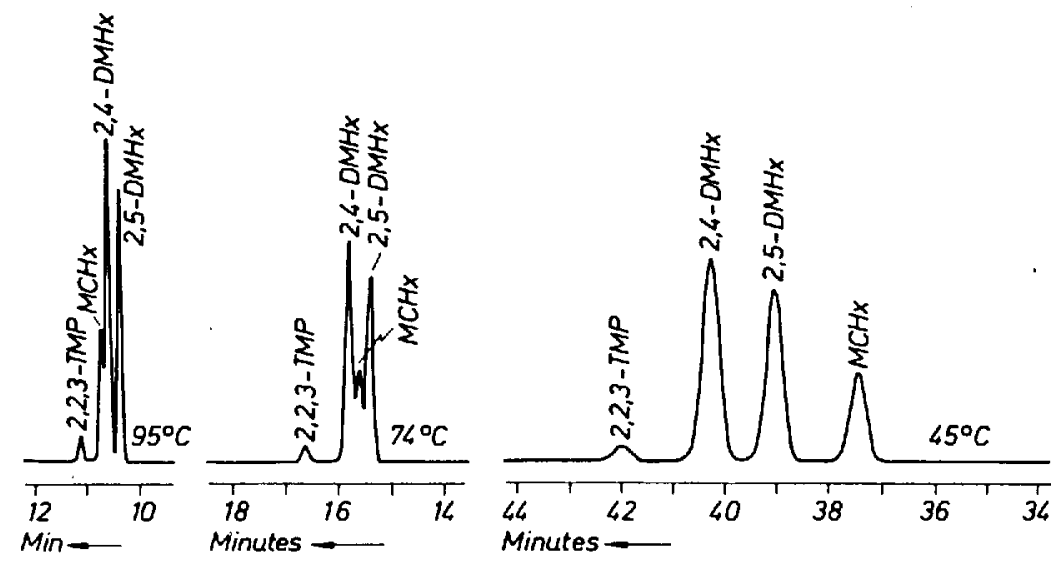

Fig. 7

- Chromatogrammes obtenues pour un mélange de méthylcyclohexane (MCHx), 2,5-diméthylhexane (2,5-DMHx), 2,4-diméthylhexane (2,4-DMHx) et 2,2,3-triméthylpentane (2,2,3-TMP) sur squalane, à trois températures différentes. Colonne $(45,7 \mathrm{~m} \times 0,5 \mathrm{~mm})$ capillaire à paroi revêtue de phase fixe liquide (squalane). Gaz-porteur, $\mathrm{He}$; vitesse linéaire moyenne: $20 \pm 1 \mathrm{~cm} / \mathrm{sec}$.
La figure 7, illustrant ce déplacement de pics, représente les trois chromatogrammes réellement obtenus, respectivement à 45,74 et $95^{\circ} \mathrm{C}$.

L'application pratique de ce déplacement de pics est, par exemple, l'essai d'identification de tels composés dans un mélange complexe d'hydrocarbures. Il suffit d'analyser l'échantillon sur une colonne renfermant du squalane (ou une autre phase fixe non polaire) à trois températures correctement choisies en fonction du domaine propre à l'échantillon et d'observer quels pics se sont "déplacés" par rapport aux autres d'une manière significative. On peut dire avec certitude que ces pics correspondent à des carbures saturés cycliques et (ou) à des carbures aromatiques.

\section{Relation entre indice de rétention et structure moléculaire}

L'indice de rétention d'un soluté peut être considéré comme une fonction correspondant à une somme de termes. Ainsi, les incréments d'indice de rétention c'est-à-dire les différences entre deux données d'indice de rétention - sont caractéristiques des groupes chimiques particuliers de la molécule de soluté ou des interactions auxquelles ils participent.

\section{Incrément correspondant à l'augmentation de l'indice de rétention pour une série d'homologues}

L'incrément d'indice de rétention le plus simple concerne les membres d'une série d'homologues analysés sur la même phase fixe liquide (et à la même température). Puisque l'échelle d'indice de rétention est équivalente à 100 fois le nombre de carbones des carbures saturés à chaine droite (par exemple 600 pour l'hexane normal, 700 pour l'heptane normal etc. ...), il est facile de comprendre que les valeurs d'indice de rétention de deux membres d'une série d'homologues, différant seulement par un groupe $\mathrm{CH}_{2}$ dans la chaîne principale, diffèrent de 100 unités d'indice. En d'autres termes, si nous appelons $I_{z}$ et $I_{(z+1)}$ les indices de rétention de deux membres consécutifs d'une série d'homologues, nous aurons:

$$
I_{(z+1)}-I_{z}=100
$$

Cette équation n'est pas valable quand $\mathrm{z}$ est inférieur à 4 ou 5 en raison du changement de pente bien connue des courbes donnant $\log t_{R}^{\prime}$ en fonction du nombre d'atomes de carbone pour les premiers membres d'une série d'homologues [26]. En pratique, une certaine déviation de la courbe par rapport à la droite pourrait aussi être trouvée pour $z>5$ mais ceci est surtout dû aux erreurs expérimentales.

La validité de l'équation 19 est illustrée par la figure 8 qui représente les courbes correspondant à un certain nombre de séries homologues d'alcanes, sur phase fixe liquide de squalane et par la figure 9 qui représente de telles courbes pour quelques séries de composés polaires sur phases fixes liquides polaires. Les données de la figure 8 proviennent de la référence [31] tandis que les données de la figure 9 proviennent de la compilation de données faites par l'ASTM [13].

La signification pratique de l'équation 19 est que, dans le chromatogramme d'un mélange complexe, on peut trouver avec une précision raisonnable les pics correspondant aux membres de certaines séries d'homologues même si on ne dispose que d'un nombre limité de données.

Incrément correspondant à l'interaction du groupe fonctionnel d'une molécule par rapport à l'hydrocarbure ayant le même nombre d'atomes de carbone

$\mathrm{Si}$, sur une phase liquide non polaire, nous analysons un composé $(x)$ contenant le groupe fonctionnel $\mathrm{R}$ et également la chaîne de l'hydrocarbure à chaine droite ayant le même nombre d'atomes de carbone, la différence entre les indices de rétention (du carbure et du composé $\mathrm{x}$ ) sera alors due à la contribution du groupe fonctionnel particulier (R) au retard chromatographique sélectif. $\mathrm{Si}$ donc, les valeurs de tels incréments d'indice de rétention sont connues, on peut prévoir la valeur de l'indice de rétention d'un composé $(\mathrm{x})$.

Prenons, par exemple, [16] les composés suivants:

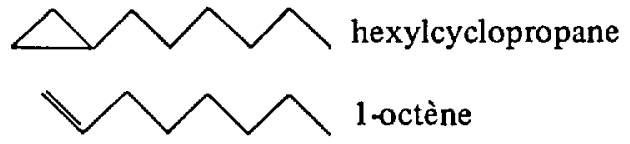

Chromatographia, Vol. 7, No. 3, March 1974 
Leurs indices de rétention*) à $80^{\circ} \mathrm{C}$ sur squalane sont respectivement 913,0 et 782,7 . Les hydrocarbures saturés à chaîne droite correspondants sont le nonane normal $(I=900)$ et l'octane normal $(I=800)$; ainsi les incréments dus aux groupes fonctionnels sont +13 et $-17,3$. Nous voulons maintenant calculer l'indice de rétention du composé suivant:

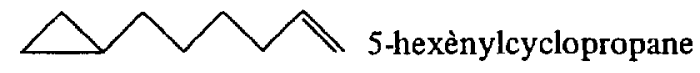

Si on suppose qu'il y a addition des effets dus aux groupes fonctionnels, l'incrément pour ce composé serait $+13,0-17,3=-4,3$. Par conséquent, l'indice de rétention calculé de ce composé est $900-4,3=895,7 . \mathrm{La}$ valeur effectivement mesurée - 895,3 - fait apparaître une excellente concordance et prouve la validité de tels calculs. Comme autre exemple, calculons l'indice de rétention du 1,7 octadiène. Puisque l'incrément pour une double liaison est de $-17,3$, il devrait être ici du double, soit $-34,6$, pour deux doubles liaisons et ainsi la valeur d'indice de rétention calculée est $800-34,6=765,4$. La valeur mesurée est 765,0 , là encore la concordance est excellente.

Le problème que posent de tels calculs est que dans les composés multifonctionnels, il y a parfois une "interaction" entre deux groupes fonctionnels et que, par conséquent, l'incrément global n'est pas toujours égal à la somme des deux incréments particuliers. Dans de tels cas, le calcul peut être affiné (rendu plus précis) en tenant compte d'incréments de second ordre traduisant cette "interaction" [16].

Mathématiquement, la valeur de l'incrément $\mathrm{H}^{\mathrm{NP}}$ peut être définie de la façon suivante:

$$
\mathrm{H}^{\mathrm{NP}}=\mathrm{I}^{\mathrm{NP}}(\mathrm{c})-\mathrm{I}^{\mathrm{NP}}(\mathrm{p})
$$

$\mathrm{I}^{\mathrm{NP}}(\mathrm{c})$ et $\mathrm{I}^{\mathrm{NP}}(\mathrm{p})$ étant respectivement les valeurs d'indice de rétention du composé (c) et du carbure saturé à chaine droite correspondant (p), sur une phase non polaire (NP).

Il faut noter que l'hydrocarbure saturé à chaine droite (p) (Eq. 20), ayant le même nombre d'atomes de carbone que (c) peut être n'importe quel hydrocarbure saturé normal utilisé dans le calcul de l'indice de rétention (Eq. 12). Par exemple, l'hexylcyclopropane $(I=913,0)$ et le 5-hexènylcyclopropane $(\mathrm{I}=895,3)$ contiennent tous les deux 9 atomes de carbone dans leur molécule et le carbure saturé à chaîne droite correspondant (p) est le nonane normal. Cependant, dans le calcul de l'indice de rétention, alors que pour l'hexylcyclopropane le nonane normal représente le carbure saturé à chaine droite à $\mathrm{z}$ atomes de carbone, pour le 5-hexènylcyclopropane il représente le carbure saturé à chaine droite à $\mathrm{z}+1$ atomes de carbone. En d'autres termes $\mathrm{I}^{\mathrm{NP}}(\mathrm{p})$ peut être inférieur ou supérieur à $I^{\mathrm{NP}}(\mathrm{c})$ et par conséquent la valeur de $\mathrm{H}^{\mathrm{NP}}$ peut être ou positive ou négative.

*) Dans la publication originale [16], les valeurs sont données avec deux décimales: nous les avons arrondies ici à une seule décimale.

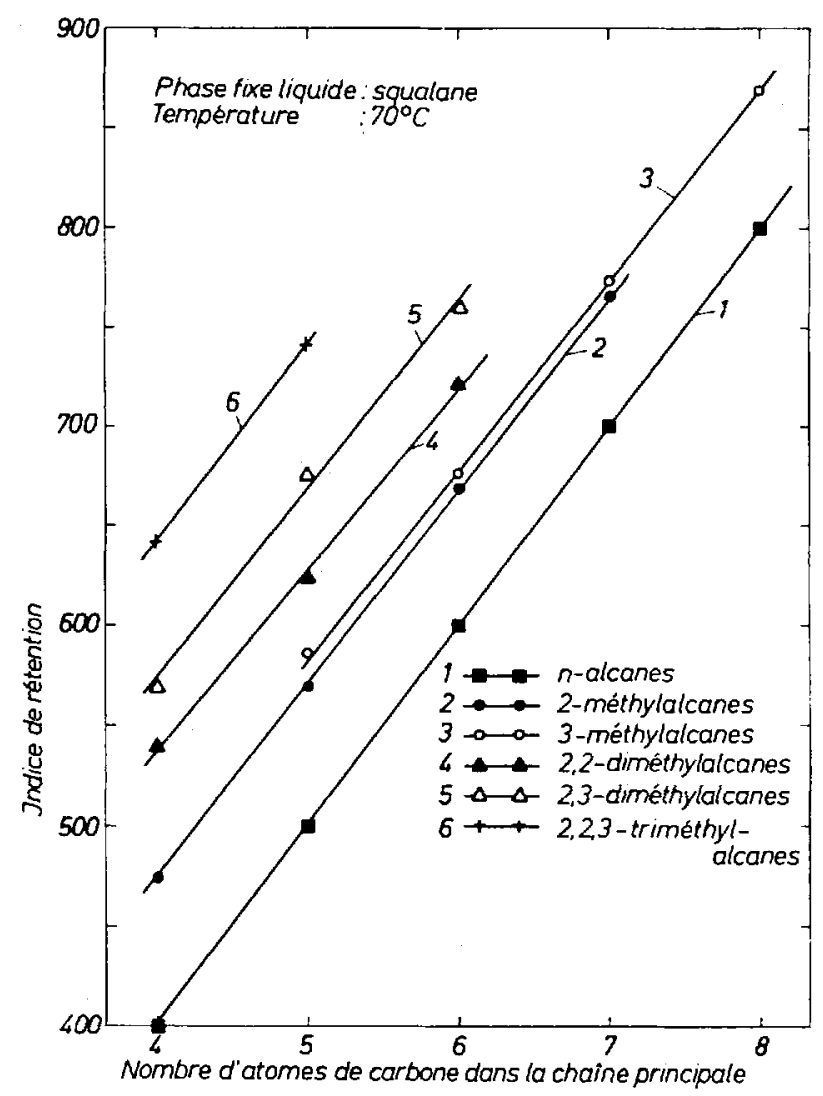

Fig. 8

- Indices de rétention sur squalane de carbures saturés, appartenant à diverses séries d'homologues, en fonction du nombre de carbone dans la chaîne principale.

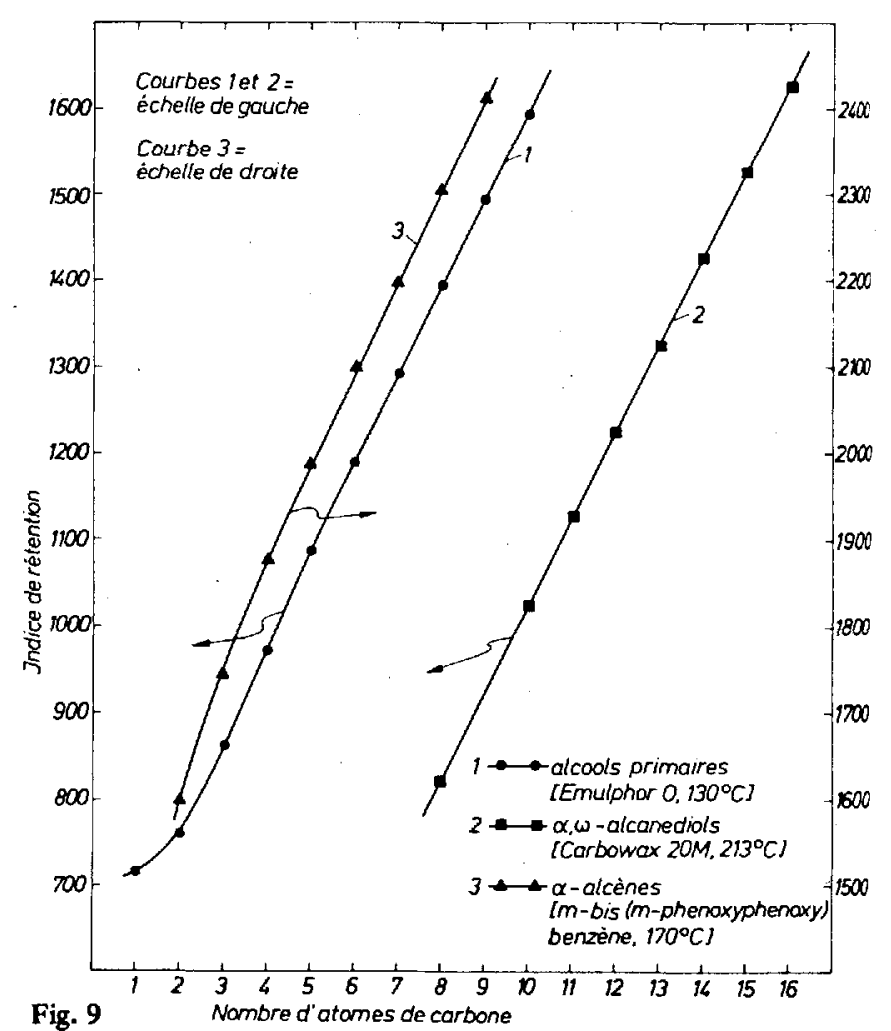

- Indices de rétention sur phase fixe liquide polaire de composés polaires, appartenant à diverses séries d'homologues, en fonction du nombre de carbone. 
Bien que, théoriquement, elle ne s'applique pas exclusivement à ce domaine, l'utilisation pratique de telles valeurs d'incréments pour prédire l'indice de rétention est limitée aux hydrocarbures puisque ces composés sont essentiellement analysés sur phases fixes liquides non polaires. C'est ainsi que Takács et al. [32, 33] ont rapporté des études détaillées relatives à la possibilité de calculer l'indice de rétention des hydrocarbures en se basant sur l'additivité des effets dus aux divers groupes fonctionnels et aux liaisons dans la molécule et en tenant compte de leurs interactions. Ils ont prouvé que ces calculs peuvent conduire à des résultats dont la précision est bonne, et plus récemment, ils se sont mis aussi à étendre le procédé à d'autres types de soluté et de phases fixes liquides [34]; cependant de nombreuses valeurs expérimentales sont nécessaires et les calculs sont longs. Une méthode d'investigation, quelque peu différente mais utilisant aussi le principe d'additivité des valeurs d'incréments et tenant compte de l'interaction des différents groupes, a été utilisée par Castello et al. [35] pour prédire la valeur de l'indice de rétention des hydrocarbures saturés à chaine ramifiée. Toutes ces études ont montré que la notion d'indice de rétention avait une base sûre, mais en raison du grand nombre de valeurs qui sont nécessaires et des équations assez compliquées que l'on doit utiliser dans les calculs, il faudra encore du temps pour que son utilité pratique soit prouvée.

\section{Incrément correspondant à l'interaction entre le groupe fonctionnel et la phase fixe liquide}

Ce type de valeur d'incrément a été présenté par Kovats dans ses publications fondamentales [3-6]. Cet incrément est similaire à celui qui vient d'être décrit sauf par le fait qu'il se rapporte au même composé mais analysé cette fois sur deux phases fixes liquides différentes dont l'une est non polaire:

$$
\Delta \mathrm{I}=\mathrm{I}^{\text {polaire }}-\mathrm{I}^{\text {nonpolaire }}
$$

Lorsqu'on utilise une phase non polaire,les solutés sortent dans l'ordre de leurs points d'ébullition tandis que la rétention sur une phase polaire dépend de l'interaction entre les groupes actifs de la molécule du soluté et les groupes actifs de la phase fixe. Ainsi, la valeur de $\Delta \mathrm{I}$ sera caractéristique de cette interaction.

En ayant choisi deux phases fixes liquides (polaire et non polaire), Kovats [3-6] a montré qu'on peut établir les valeurs d'incrément particulières attribuables aux divers groupes caractéristiques ou "zones" dans la molécule de soluté dont les influences s'additionnent pour modifier la valeur de $\Delta I$ observée dans le cas d'un soluté donné. Ainsi, connaissant les valeurs d'incrément particulières, on peut calculer la valeur de $\Delta \mathrm{I}$ prévisible pour un soluté donné. On peut, par ce moyen, établir l'identité d'un certain pic en comparant la valeur de $\Delta I$ mesurée aux valeurs calculées pour différentes structures possibles.

Il est plus facile d'illustrer cette possibilité par des exemples réels que d'en discuter en termes généraux. Notre premier exemple concerne les quatre alcools butyliques isomères. Nous avons analysé leur mélange sur une colonne renfermant de l'Apiézon L et une autre de l'Emulphor $\mathrm{O}$ et nous avons calculé pour chaque pic de soluté la valeur de $\Delta \mathrm{I}$; ces valeurs sont reportées au tableau $V$. Nous voudrions maintenant assigner à chaque pic d'alcool en $\mathrm{C}_{4}$ le nom qui lui correspond. Pour cela, nous utilisons les valeurs d'incrément publiées par Wehrli et Kovats [6] pour ces deux phases fixes liquides particulières: celles-ci sont partiellement reproduites au tableau VI et ici, nous sommes intéressés par les valeurs correspondant aux alcools.

En utilisant les valeurs données dans le tableau VI, nous pouvons calculer la valeur de $\Delta I$ prévisible pour chacun des quatre alcools butyliques. La valeur de base est, dans tous les cas, 453 et les valeurs d'incrément à retrancher sont composées de la manière qui est indiquée ci-dessous pour les quatre cas:

$$
\begin{array}{cccc}
\text { alcool butylique primaire }=\text { Butane, } 1-01 & \Delta I=453-80=373 \\
\mathrm{CH}_{3}-\mathrm{CH}_{2}-\mathrm{CH}_{2}-\mathrm{C}-\mathrm{OH} & \mathrm{R}_{1}=\mathrm{C}_{3} & \ldots \ldots \ldots, & -80 \\
\text { I } & \mathrm{R}_{2}=\mathrm{H} & \ldots \ldots \ldots & 0 \\
\mathrm{H} & \mathrm{R}_{3}=\mathrm{H} & \ldots \ldots \ldots & \frac{0}{-80}
\end{array}
$$

alcool butylique secondaire $=$ Butane, $2-01 \quad \Delta I=453-133=320$

$$
\begin{array}{ccccc}
\mathrm{H} & & & \\
\mathrm{CH}_{3}-\mathrm{CH}_{2}-\mathrm{C}-\mathrm{CH}_{3} & \mathrm{R}_{1}=\mathrm{C}_{2} & \ldots \ldots \ldots & -76 \\
\text { I } & \mathrm{R}_{2}=\mathrm{C}_{1} & \ldots \ldots \ldots & -57 \\
\mathrm{OH} & \mathrm{R}_{3}=\mathrm{H} & \ldots \ldots \ldots & \frac{0}{-133}
\end{array}
$$

alcool isobutylique $=2-\mathrm{Méthyl}$, $\Delta \mathrm{I}=453-95=358$ n-propane, 1-ol<smiles>CC(C)CO</smiles>

$$
\begin{array}{llr}
R_{1}=C_{2} & \ldots \ldots \ldots & -76 \\
R_{2}=H & \ldots \ldots & 0 \\
R_{3}=H & \ldots \ldots \ldots & 0 \\
C_{1} \text { à } \alpha \text { sur } R_{1} \ldots . & -19 \\
\end{array}
$$

alcool butylique tertiaire = 2-Méthyl, $\Delta \mathrm{I}=453-171=282$ n-propane, 2-ol<smiles>CC(C)(C)O</smiles>

$$
\begin{aligned}
& \mathrm{R}_{1}=\mathrm{C}_{1} \ldots \ldots \ldots \ldots-57 \\
& R_{2}=C_{1} \ldots \ldots \ldots-57 \\
& \mathrm{R}_{3}=\mathrm{C}_{1} \quad \cdots \cdots \cdots \cdot \frac{-57}{-171}
\end{aligned}
$$

Dans la partie droite du tableau $V$, on compare les valeurs calculées aux valeurs obtenues par mesure et, en conséquence, on assigne à chaque pic un nom de composé. Comme on peut le voir, la concordance est bonne. Naturellement, nous ne pouvons pas attendre une parfaite concordance entre les valeurs calculées et mesurées et on doit estimer satisfaisante une concordance à quelques unités d'indice près.

Ce calcul montre aussi l'importance non seulement de la nature du groupe fonctionnel mais aussi de la position qu'il occupe. En d'autres termes, on ne pourrait pas avoir simplement une valeur d'incrément pour le groupe hy- 
Tableau V. Résultats obtenus pour les quatre alcools butyliques isomères ${ }^{\mathrm{a}}$

\begin{tabular}{l|c|c|c|c|l}
\hline \multirow{2}{*}{ Pics } & \multicolumn{2}{|l|}{ Indices de rétention mesurés } & $:$ & & substances \\
\cline { 2 - 3 } & Apiézon L & Emulphor O & $\begin{array}{c}\Delta \mathrm{I} \\
\text { mesurés }\end{array}$ & $\begin{array}{c}\Delta \mathrm{I} \\
\text { calculés }\end{array}$ & \\
\hline A & 472 & 758 & 286 & 282 & 2-méthyl, n-propane, 2-ol \\
B & 553 & 871 & 318 & 320 & n-butane, 2-ol \\
C & 575 & 929 & 354 & 358 & 2-méthyl, n-propane, 1-ol \\
D & 606 & 973 & 367 & 373 & n-butane, 1-ol \\
\hline
\end{tabular}

a mesurés à $130^{\circ} \mathrm{C}$

droxyle mais on doit aussi tenir compte de "l'interaction" entre le groupe actif et les autres groupes présents dans la molécule.

Donnons encore un exemple tiré de la publication de Wehrli et Kovats [6]. Au cours de l'étude d'huiles essentielles, on a trouvé un alcool terpénique mais on n'était pas certain de sa structure. Nous donnons ci-dessous les quatre structures possibles ainsi que les valeurs calculées de $\Delta \mathrm{I}$ correspondant à ces structures:<smiles>CC(C)(O)C1CC=C(I)CC1</smiles><smiles>CC(C)C1(O)CC=CCC1</smiles><smiles>CC1=CC(O)C(C([Si])[Si])CC1</smiles><smiles>OC1([W])CC=C(C([Al])[Se])CC1</smiles>

En calculant l'indice de rétention du pic en question, observé à la sortie des deux colonnes préparées l'une avec l'Emulphor $O$, l'autre avec l'Apiézon $\mathrm{L}$ comme phases fixes liquides, on a obtenu pour $\Delta \mathrm{I}$ une valeur de 270. Comme on peut le voir, la valeur calculée pour la structure II est la plus proche de la valeur mesurée et c'est donc cette structure qui a été admise. Des études ultérieures de CG-SM ont confirmé cette identification.

\section{Constante de Rohrschneider}

Se basant sur le travail de Kovats, Rohrschneider a, en 1965-1966, proposé de faire un pas de plus dans la signification et l'utilisation de l'indice de rétention et la notion d'incrément $[36,37]$. Il a estimé que les incréments d'indice de rétention $\Delta I$ sont formés de termes additifs qui résultent, eux-mêmes, de deux groupes de facteurs: les uns spécifiques du soluté (échantillon) qui restent inchangés quand change la phase fixe liquide et les autres spécifiques de la phase fixe liquide et qui ne dépendent pas du soluté mais se rapportent à un nombre limité de substances-témoins sélectionnées. Les premiers sont désignés par les symboles $a, b, c, d$ et e et les seconds par les symboles*) x, y, z, u et s. Par définition, l'incré-

\footnotetext{
*) Dans sa première publication [36], Rohrschneider pensait que trois substances suffisaient et utilisait les symboles $x$, y et $z$ pour exprimer les trois constantes correspondantes, spécifiques de la phase fixe liquide, se rapportant à ces trois substances témoins. Quand, un an plus tard, il ajouta deux substances témoins supplémentaires aux trois déjà choisies [37\}, il ne voulut pas modifier les symboles des constantes déjà existantes. C'est pour cette raison que les symboles ne suivent pas l'ordre alphabétique.
}

ment d'indice de rétention $\Delta \mathrm{I}$ d'un soluté, sur une phase fixe donnée est exprimé comme:

$$
\Delta \mathrm{I}=\mathrm{ax}+\mathrm{by}+\mathrm{cz}+\mathrm{du}+\mathrm{es}
$$

Voyons quelle est la signification de ces constantes. Constantes spécifiques de la phase fixe. Elles sont obtenues en sélectionnant cinq substances tẻmoins et en déterminant pour chacune la valeur de $\Delta \mathrm{I} / 100$. Par exemple, pour le benzène qui est la première substance témoin

$\mathrm{x}=\frac{\Delta \mathrm{I}(\text { benzene })}{100}=\frac{\mathrm{I}^{\text {phase }}(\text { benzene })-\mathrm{I}^{\text {squalane }}(\text { benzene })}{100}$

Les autres substances témoins proposées par Rohrschneider sont l'éthanol, la méthyléthylcétone, le nitrométhane et la pyridine et les symboles pour les valeurs de $\Delta \mathrm{I} / 100$ sont respectivement $y, z$, $u$ et $s$. Chaque substance témoin est représentative des interactions d'un groupe donné (présent dans l'échantillon) avec une phase fixe liquide donnée et nous traiterons ultérieurement de cette question dans la troisième partie de cette série.

Constantes spécifiques du soluté. Ainsi qu'il a été indiqué précédemment, celles-ci-caractérisent uniquement un soluté donné (échantillon) et sont indépendantes de la phase fixe liquide. On peut les établir en déterminant les constantes spécifiques de phases fixes liquides pour au moins cinq phases liquides, puis en mesurant la valeur de $\Delta \mathrm{I}$ pour le soluté considéré sur les cinq phases (ou plus) par rapport au squalane, et en résolvant finalement les équations pour les constantes $\mathbf{a}, \ldots$ e:

$$
\begin{aligned}
& I^{\text {Ph.1 }}-I^{\text {squalane }}=a_{1}+b y_{1}+c z_{1}+d u_{1}+e s_{1} \\
& I^{\text {Ph.2 }}-I^{\text {squalane }}=a x_{2}+b y_{2}+c z_{2}+d u_{2}+e s_{2} \\
& \vdots \\
& I^{\text {Ph.5 }}-I^{\text {squalane }}=a_{5}+b y_{5}+c z_{5}+d u_{5}+e s_{5}
\end{aligned}
$$

dans lesquelles les indices $1 \ldots 5$ des constantes spécifiques de phase se rapportent aux cinq phases. Pour le traitement mathématique complet, voir la publication de Rohrschneider [37].

Nous avons vu que les constantes spécifiques de phase $\mathrm{x}$... s représentent les valeurs $\Delta \mathrm{I} / 100$ pour la substance témoin sur cette phase. Ainsi, il est facile de comprendre que les constantes a ... e des cinq substances témoins utilisées pour déterminer les constantes spécifiques de phase sont posées par définition. On en trouvera la liste au tableau VII. 
Tableau VI. Accroissements d'indices de rétention utilisés pour le calcul de $\Delta I$ (termes ou "incréments" caractéristiques de chaque groupe chimique)

Phases fixes liquides: polaire, Emulphor $\mathrm{O}$

non polaire, Apiézon L

\begin{tabular}{|c|c|c|c|c|c|c|c|c|c|c|}
\hline \multirow{3}{*}{$\begin{array}{l}\text { Type de } \\
\text { substance }\end{array}$} & \multirow{3}{*}{$\begin{array}{c}\text { substituant } \\
\mathrm{X}\end{array}$} & \multirow{3}{*}{ Famille } & \multirow{3}{*}{$\begin{array}{l}\text { Valeur } \\
\text { de base }\end{array}$} & \multicolumn{4}{|c|}{$\mathbf{R}=$} & \multirow{2}{*}{\multicolumn{3}{|c|}{$\begin{array}{l}\text { Groupe } \\
\text { méthyl sur R } \\
\text { en position }\end{array}$}} \\
\hline & & & & \multirow{2}{*}{$c_{1}$} & \multirow{2}{*}{$C_{2}$} & \multirow{2}{*}{$\mathrm{C}_{3}$} & \multirow{2}{*}{$\begin{array}{l}\mathrm{C}_{4} \text { et } \\
\text { supérieur }\end{array}$} & & & \\
\hline & & & & & & & & $\alpha$ & $\beta$ & $\gamma$ \\
\hline \multirow{3}{*}{$\mathbf{R}_{\mathbf{2}}-\mathrm{C}-\mathrm{X}$} & $\mathrm{OH}$ & alcool & 453 & -57 & -76 & -80 & -82 & -19 & -4 & -2 \\
\hline & $\mathrm{Cl}$ & chlorure & 174 & -21 & -33 & -39 & -44 & -12 & -6 & -5 \\
\hline & $\mathrm{Br}$ & bromure & 184 & -21 & -33 & -39 & -44 & -12 & -6 & -5 \\
\hline
\end{tabular}

Bien que les constantes spécifiques de phase et les constantes spécifiques de soluté aient été proposées par Rohrschneider, en pratique, le terme "constantes de Rohrschneider" est appliqué automatiquement aux constantes spécifiques de phase. La raison en est que l'utilisation du concept de Rohrschneider pour la caractérisation du soluté, et la prévision de la valeur de $\Delta \mathrm{I}$ pour un soluté donné sur une phase donnée est assez compliquée et rend nécessaire la possession de données qui ne sont pas facilement disponibles; par ailleurs, l'utilisation des constantes spécifiques de phase pour la caractérisation d'une phase fixe liquide est de plus en plus répandue grâce, principalement, au travail de Supina et Rose [38] et de McReynolds [18]. Ceci sera le sujet de la troisième partie de cette série d'articles.

Tableau VII. Constantes de Rohrschneider caractéristiques du soluté pour les cinq substances de référence

\begin{tabular}{lccc|c|c|c}
\hline & $\mathrm{a}$ & $\mathrm{b}$ & $\mathrm{c}$ & $\mathrm{d}$ & $\mathrm{e}$ \\
\hline benzène & 100 & 0 & 0 & 0 & 0 \\
éthanol & 0 & 100 & 0 & 0 & 0 \\
méthyléthylcétone & 0 & 0 & 100 & 0 & 0 \\
nitrométhane & 0 & 0 & 0 & 100 & 0 \\
pyridine & 0 & 0 & 0 & 0 & 100 \\
\hline
\end{tabular}

\section{Corrélation entre indice de rétention et rétention relative}

La chromatographie en phase gazeuse étant une technique de séparation, la question essentielle, lorsque nous voulons analyser un mélange donné, est de savoir si les composés A et $B$ peuvent être séparés dans certaines conditions expérimentales (phase fixe liquide, température) sur une colonne donnée. Nous avons déjà donné (première partie, équation 6) l'équation de base reliant le degré (facteur) de résolution de deux pics (R) de solutés, la rétention relative $(\alpha)$ pour ce couple de solutés et le facteur de capacité (rapport de distribution) (k) du soluté du second pic, à l'efficacité de la colonne (nombre de plateaux théoriques $\mathbf{n}$ ).

$$
n=16 R^{2}\left(\frac{\alpha}{\alpha-1}\right)^{2}\left(\frac{k_{2}+1}{k_{2}}\right)^{2}
$$

On peut tirer de l'équation 6 le degré (facteur) de résolution $(\mathrm{R})$ de deux pics:

$$
\mathrm{R}=\frac{\sqrt{\mathrm{n}}}{4}\left(\frac{\alpha-1}{\alpha}\right)\left(\frac{\mathrm{k}_{2}}{\mathrm{k}_{2}+1}\right)
$$

En se basant sur sa connaissance de la chromatographie en phase gazeuse, un expérimentateur pourra, d'après les valeurs de rétention relative, se rendre compte de la facilité ou de la difficulté des problèmes de séparation. Par exemple, deux pics de solutés pour lesquels $\alpha>1,10$ peuvent habituellement être séparés avec une colonne convenablement remplie alors que, si $\alpha<1,05$, la séparation est difficile même avec de longues colonnes capillaires. Ainsi pour établir les conditions d'analyse, on devrait avoir une idée de la rétention relative à laquelle on peut s'attendre pour la paire de pics de solutés dont la séparation est la plus difficile et naturellement la question se pose: pouvons-nous estimer la rétention relative à partir de l'indice de rétention?

Traitons brièvement de cette question; pour un exposé détaillé voir la référence [39].

Si nous considérons deux pics adjacents correspondant aux composés $n$ et $m$ - pour lesquels $t_{\mathrm{R}(\mathrm{m})}^{\prime}>\mathrm{t}_{\mathrm{R}(\mathrm{n})}^{\prime}$ et donc $I_{m}>I_{n}-$ qui se placent entre les pics des deux hydrocarbures à chaine droite correspondant à la définition de l'indice de rétention, nous pouvons alors exprimer la différence des deux indices de rétention, $i$, de la manière suivante:

$$
\begin{aligned}
i=I_{m}-I_{n} & =100 \frac{\log t_{R(m)}^{\prime}-\log t_{R(n)}^{\prime}}{\log t_{R(z+1)}^{\prime}-\log t_{R(z)}^{\prime}} \\
& =100 \frac{\log \alpha_{m / n}}{\log r_{(z+1) / z}}
\end{aligned}
$$

Equation dans laquelle:

$$
\begin{aligned}
\alpha_{m / n} & =t_{R(m)}^{\prime} / t_{R(n)}^{\prime} \\
r_{(z+1) / z} & =t_{R(z+1)}^{\prime} / t_{R(z)}^{\prime}
\end{aligned}
$$

Ainsi que nous l'avons déjà expliqué dans la première partie, les symboles $\alpha$ et $r$ expriment tous les deux des rétentions relatives, la seule différence étant que $\alpha$ exprime toujours la rétention relative de deux pics consécutifs. 
Nous avons déjà vu dans la première partie que, pour une température et une phase fixe liquide données, $r_{(z+1) / z}$ est constant et indépendant de $z$. Donc $100 / \log r_{(z+1) / z}$ est également constant et nous pouvons écrire l'équation 25 de la manière suivante:

$$
\mathrm{i}=\text { (constante) } \log \alpha_{\mathrm{m} / \mathrm{n}}
$$

ou

$$
\log \alpha_{\mathrm{m} / \mathrm{n}}=\text { (constante) } \mathrm{i}
$$

On peut facilement déterminer la rétention relative de deux hydrocarbures saturés à chaîne droite consécutifs à une température quelconque, sur une phase fixe donnée quelconque et des tables ou courbes donnant ces variations peuvent être préparées; disposant ainsi de cette valeur, on peut calculer à partir de la différence des deux indices de rétention, la rétention relative de deux pics adjacents à séparer et, si on possède des données concernant les performances de la colonne, on peut aussi déterminer si une colonne donnée convient à la séparation.

On peut même aller plus loin dans la simplification de l'équation $28 \mathrm{~b}$. Une règle mathématique connue dit que pour les faibles valeurs de $\chi, \log (1+\chi)$ est proportionnel à $\chi$. En d'autres termes:

$$
\chi \simeq \text { (constante) } \mathbf{i}
$$

pour

$$
\chi=\alpha-1
$$

Ceci signifie par exemple que si $\alpha$ passe de 1,05 à 1,10 , il en résultera un changement de valeur de i d'environ $100 \%$. Par exemple, sur le squalane, à $100^{\circ} \mathrm{C}$, les valeurs sont respectivement:

$$
\begin{array}{ll}
\alpha=1,05 & \mathrm{i}=6,5 \\
\alpha=1,10 & \mathrm{i}=12,5
\end{array}
$$

Puisque les valeurs d'indice de rétention sont habituellement arrondies au nombre entier le plus voisin, le calcul de i ne donnera que des valeurs approchées. Celles-ci sont cependant assez proches des valeurs réelles et puisque de tels calculs ne sont utilisés que pour déterminer les conditions convenables d'analyse, les écarts qui en résultent n'ont pas d'influences sérieuses.

L'exemple donné ci-dessous illustre l'utilisation de i pour un calcul pratique [39].

Nous voudrions savoir quel genre de résolution nous pouvons attendre d'une colonne capillaire de 45,72 mètres ( 150 pieds) de long avec le squalane comme phase fixe liquide à $45^{\circ} \mathrm{C}$ pour les deux pics de 2,5 diméthylhexane et du 2,4-diméthylhexane. A cette température, notre colonne a une valeur de HETP de $0,77 \mathrm{~mm}$ et un rapport de distribution (facteur de capacité) (k) de 9,12 pour le 2,4-DMHx, et les valeurs d'indice de rétention sont respectivement:

$$
\text { 2,5-diméthylhexane } 729
$$$$
\text { 2,4-diméthylhexane } 732,5
$$

Donc, $\mathrm{i}=732,5-729=3,5$.
Nous avons établi précédemment que, à $45^{\circ} \mathrm{C}, \mathrm{r}_{(\mathrm{z}+1) / \mathrm{z}}$ $=2,745$. Nous pouvons donc calculer $\alpha$ à partir de l'équation 25:

$$
\log \alpha=\frac{3,5(\log 2,745)}{100}=0,01314
$$

et, ainsi, $\alpha=1,036$.

La relation existant entre la valeur de $\operatorname{HETP}(\mathrm{h})$, la longueur de la colonne (L) et le nombre de plateaux théoriques $(\mathrm{n})$ a déjà été exprimée dans l'équation 3 :

$$
\mathrm{n}=\frac{\mathrm{L}}{\mathrm{h}}
$$

Puisque $\mathrm{h}=0,77 \mathrm{~mm}$ et $\mathrm{L}=150$ pieds $=45,72$ mètres, $\mathrm{n}=59,400$. En reportant ces valeurs dans l'équation $6 \mathrm{a}$, nous obtenons un degré de résolution $R=1,91$.

Nous avons vérifié ce calcul en analysant l'échantillon sur la colonne et nous avons obtenu $\alpha=1,034$ et $R=1,84$. Cette concordance est très bonne.

\section{Références}

[23] J. K. Haken, J. Chromatog. Sci. 11, 144-150 (1973).

[24] L. Rohrschneider, J. Chromatog. Sci. 11, 160-166 (1973).

[25] R. A. Keller, J. Chromatog. Sci. 11, 188-190 (1973).

[26] J. M. Takács, J. Chromatog. Sci. 11, 210-215 (1973).

[27] P. Chovin and J. Lebbe, in "Journées Internationales d'Etude des Méthodes de Séparation Immédiate et de Chromatographi ed. J. Tranchant, G.A.M.S., Paris 1961; pp. 90-103.

[28] P. Chovin and J. Lebbe, J. Gas Chromatog. 4, 37-41 (1966).

[29] J. Takács, M. Rockenbauer and I. Olácsi, J. Chromatog. 42 19-28 (1969).

[30] E. B. Molnár, P. Moritz and J. Takács, J. Chromatog. 66, 205-212 (1972).

[31] Data Subcommittee of the GC Discussion Group, J. Gas Chromatog. 4, 1-3 (1966).

[32] J. Takács, M. Rockenbauer and I. Olácsi, J. Chromatog. 66, 19-28 (1969).

[33] J. Takács, Zs. Tálas, I. Bernát, Gy. Czakó and A. Fischer, J. Chromatog. 67, 203-212 (1972).

[34] J. M. Takács, E. Kocsi, E. Garamvölgyi, E. Eckhart, T. Lombosi, Sz. Nyiredy Jr., I. Borbély and Gy. Krasznai, J. Chromatog. 81, 1-8 (1973).

[35] G. Castello, M. Lunardelli and M. Berg, J. Chromatog. 76, 31-44 (1973).

[36] L. Rohrschneider, J. Chromatog. 17, 1-12 (1965).

[37] L. Rohrschneider, J. Chromatog. 22, 6-22 (1966).

[38] W. R. Supina and L. R. Rose, J. Chromatog. Sci. 8, 214-217 (1970).

[39] L. S. Ettre and K. Billeb, J. Chromatog. 30, 12-16 (1967). 